\title{
Comparing the potential of MEG and EEG to uncover brain tracking of speech temporal envelope
}

Florian Destoky $^{1}$, Morgane Philippe ${ }^{1}$, Julie Bertels ${ }^{1,2}$, Marie Verhasselt ${ }^{1}$, Nicolas Coquelet ${ }^{1}$, Marc Vander Ghinst ${ }^{1}$, Vincent Wens ${ }^{1,3}$, Xavier De Tiège ${ }^{1,3}$, Mathieu Bourguignon ${ }^{1,4,5}$

${ }^{1}$ Laboratoire de Cartographie fonctionnelle du Cerveau, UNI - ULB Neuroscience Institute, Université libre de Bruxelles (ULB), Brussels, Belgium.

${ }^{2}$ Consciousness, Cognition and Computation group, UNI - ULB Neuroscience Institute, Université libre de Bruxelles (ULB), Brussels, Belgium.

${ }^{3}$ Department of Functional Neuroimaging, Service of Nuclear Medicine, CUB Hôpital Erasme, Université libre de Bruxelles (ULB), Brussels, Belgium.

${ }^{4}$ Laboratoire Cognition Langage et Développement, UNI - ULB Neuroscience Institute, Université libre de Bruxelles (ULB), Brussels, Belgium.

${ }^{5}$ BCBL, Basque Center on Cognition, Brain and Language, 20009 San Sebastian, Spain.

\section{Corresponding author}

Florian Destoky, Laboratoire de Cartographie fonctionnelle du Cerveau, UNI - ULB Neuroscience Institute, Université libre de Bruxelles, 808 Lennik Street, 1070 Brussels, Belgium. E-mail: florian.destoky@ulb.ac.be. Tel. +32 25553286.

Number of tables: 2, number of figures: 8 , number of pages: 49 , number of words in the Abstract: 360, number of words in the main text: 9041 


\section{Conflicts of interest}

None of the authors disclose any potential conflict of interest.

\section{Highlights}

- Speech brain tracking was analyzed from simultaneous MEG and EEG data.

- Uncovering speech brain tracking requires 3 times shorter MEG than EEG recordings.

- Some previous MEG findings were replicated with MEG but not with EEG.

\section{Acknowledgments}

Florian Destoky, Julie Bertels, and Mathieu Bourguignon are supported by the program Attract of Innoviris (grant 2015-BB2B-10). Marc Vander Ghinst was supported by a research grant from the Fonds Erasme (Brussels, Belgium). Xavier De Tiège is Post-doctorate Clinical Master Specialist at the FRS-FNRS. Mathieu Bourguignon is supported by the Marie Skłodowska-Curie Action of the European Commission (grant 743562) and by the Spanish Ministry of Economy and Competitiveness (grant PSI2016-77175-P). This research project and the MEG project at the CUB Hôpital Erasme are financially supported by the Fonds Erasme (Research convention “Les Voies du Savoir”, Fonds Erasme, Brussels, Belgium). 


\begin{abstract}
:
During connected speech listening, brain activity tracks speech rhythmicity at delta $(\sim 0.5 \mathrm{~Hz})$ and theta $(4-8 \mathrm{~Hz})$ frequencies. Here, we compared the potential of magnetoencephalography (MEG) and high-density electroencephalography (EEG) to uncover such speech brain tracking.
\end{abstract}

Ten healthy right-handed adults listened to two different 5-min audio recordings, either without noise or mixed with a cocktail-party noise of equal loudness. Their brain activity was simultaneously recorded with MEG and EEG. We quantified speech brain tracking channel-by-channel using coherence, and with all channels at once by speech temporal envelope reconstruction accuracy.

In both conditions, speech brain tracking was significant at delta and theta frequencies and peaked in the temporal regions with both modalities (MEG and EEG). However, in the absence of noise, speech brain tracking estimated from MEG data was significantly higher than that obtained from EEG. Furthemore, to uncover significant speech brain tracking, recordings needed to be $\sim 3$ times longer in EEG than MEG, depending on the frequency considered (delta or theta) and the estimation method. In the presence of noise, both EEG and MEG recordings replicated the previous finding that speech brain tracking at delta frequencies is stronger with attended speech (i.e., the sound subjects are attending to) than with the global sound (i.e., the attended speech and the noise combined). Other previously reported MEG findings were replicated based on MEG but not EEG recordings: 1) speech brain tracking at theta frequencies is stronger with attended speech than with the global sound, 2) speech brain tracking at delta frequencies is stronger in noiseless than noisy conditions, and 3) when noise is added, speech brain tracking at delta frequencies dampens less in the left hemisphere than in the the right hemisphere. Finally, sources of speech brain 
tracking reconstructed from EEG data were systematically deeper and more posterior than those derived from MEG.

The present study demonstrates that speech brain tracking is better seen with MEG than EEG. Quantitatively, EEG recordings need to be $\sim 3$ times longer than MEG recordings to uncover significant speech brain tracking. As a consequence, MEG appears more suited than EEG to pinpoint subtle effects related to speech brain tracking in a given recording time.

Keywords: speech brain tracking, MEG, EEG, reconstruction accuracy, coherence 


\section{Introduction}

Social interactions are of tremendous importance in human society and largely rely on verbal language interactions. Still, the neural mechanisms allowing the human brain to decode speech signals in real time are poorly understood (Zion-Golumbic et al., 2012). Based on electroencephalographic (EEG) or magnetoencephalographic (MEG) recordings, it was shown that listerner's auditory cortices track the time course of speaker's speech temporal envelope at $0.5 \mathrm{~Hz}$ (delta frequencies) and 4-8 Hz (theta frequencies) (Ahissar et al., 2001; Bourguignon et al., 2013; Broderick et al., 2017; Di Liberto et al., 2015; Ding et al., 2017; Ding and Simon, 2014; Gross et al., 2013; Horton et al., 2013; Keitel et al., 2018; Kösem and van Wassenhove, 2016; Luo and Poeppel, 2007; Meyer and Gumbert, 2018; Molinaro et al., 2016; Müller et al., 2018; O’Sullivan et al., 2014; Peelle et al., 2013; Pellegrino et al., 2011; Puschmann et al., 2017; Zion-Golumbic et al., 2012). As delta and theta frequencies match with phrasal/sentence and syllable repetition rates respectively, it has been hypothesized that corresponding brain oscillations subserve the chunking of incoming speech into relevant segments for further speech recognition (Ahissar et al., 2001). In line with this view, speech brain tracking is stronger when listening to intelligible speech compared to non-intelligible speech (Ahissar et al., 2001; Luo and Poeppel, 2007; Peelle et al., 2013; Riecke et al., 2018), and delta fluctuations track sentence boundaries, even in the absence of prosodic cues (Ding et al., 2016; Meyer et al., 2016).

Studies on speech brain tracking have also increased our understanding of the neural mechanisms subtending speech perception in adverse listening conditions (Ding and Simon, 2014; O'Sullivan et al., 2014; Riecke et al., 2018; Zion-Golumbic et al., 2013; Zion-Golumbic and Schroeder, 2012). Indeed, in cocktail-party conditions, 1) speech brain tracking at delta and theta frequencies is stronger with the attended speech (i.e., the sound 
subjects are attending to) than with the global sound (i.e., the attended speech and the noise combined) (Broderick et al., 2017; Ding and Simon, 2012; Fuglsang et al., 2017; Horton et al., 2013; Luo and Poeppel, 2007; Mesgarani and Chang, 2012; O’Sullivan et al., 2014; Puschmann et al., 2017; Rimmele et al., 2015; Simon, 2015; Vander Ghinst et al., 2016; Zion-Golumbic et al., 2013), 2) it decreases when the signal-to-noise ratio (SNR) decreases (Giordano et al., 2016; Vander Ghinst et al., 2016; but see Ding and Simon, 2013 who found that speech brain tracking remains stable as long as speech is intelligible), 3) this dampening is more stringent in the right (vs. left) hemisphere at delta frequencies (Vander Ghinst et al., 2016), and 4) high-order brain regions track only the attended speech stream with no detectable trace of the unattended speech (Zion-Golumbic et al., 2013).

In addition to its interest for the basic understanding of speech processing, speech brain tracking holds the promise of enabling the development of novel impactful applications. For example, speech brain tracking could be used as a tool for pre-surgical localization of language brain functions. It could be used for objective assessment of hearing functions in individuals with developmental hearing loss, to evaluate how their condition impacts their brain functions (Petersen et al., 2017), and to determine if they would benefit from a cochlear implant. Finally, speech brain tracking in noisy conditions could be used to assess auditory attentional selection functions (O’Sullivan et al., 2014; Rimmele et al., 2015; Zion-Golumbic et al., 2012) in individuals presenting a disorder of consciousness (Laureys et al., 2004; Owen et al., 2006).

Only two non-invasive techniques afford a temporal resolution high enough to properly estimate speech brain tracking: MEG and EEG. Although tightly related, these two techniques differ largely on several aspects. MEG is mainly sensitive to primary currents while EEG measures only the volume conduction currents (Hari and Puce, 2017). MEG has a 
heightened sensitivity to currents tangential to the skull and is mainly blind to deep sources while EEG measures voltage fluctuations arising from sources in all orientations, and sees deep sources better (Ahlfors et al., 2010; Hari and Puce, 2017). This implies that EEG electrodes are sensitive to larger brain volumes than MEG sensors, especially compared to planar gradiometers. Consequently, scalp distributions are more widespread in EEG than MEG, which makes it difficult, for instance, to discriminate with EEG the contribution of left and right auditory cortices to the auditory N2 component (Crowley and Colrain, 2004; Pereira et al., 2014). On the other hand, the cost of MEG in its current form is a tremendous limitation to its large scale usability.

Despite the profusion of studies on speech brain tracking conducted with MEG or EEG recordings, the potential of these two recording modalities to uncover significant speech brain tracking has not yet been compared. The outcome of such comparison would undoubtedly help researchers and, in a foreseeable future, clinicians determine which modality they should opt for to properly investigate speech brain tracking.

Here, we compare the potential of MEG and high-density EEG (256 electrodes) to uncover significant speech brain tracking based on simultaneous recordings. We also test the hypothesis that speech brain tracking can be observed with equal accuracy based on both modalities, provided that adequate channel combination is used. Finally, we illustrate the impact of the choice of the recording modality to identify relevant effects. Specifically, we evaluate whether the effects highlighted above that concern speech listening in noisy conditions can be replicated based on MEG and EEG recordings. 


\section{Methods}

Unless stated otherwise, data analyses were performed using custom-made Matlab scripts. These scripts as well as fully anonymized data are available upon reasonable request to the corresponding author.

\subsection{Subjects}

Ten healthy adult human subjects (5 women) took part in this experiment. They were aged $25 \pm 4$ years (mean \pm SD) and native French speakers. None of them had prior developmental, neurological, or psychiatric disorder. All subjects had normal hearing according to pure tone audiometry (i.e., normal hearing thresholds (between 0-20 dB HL) for 250, 500, 1000, 2000, 4000 and $8000 \mathrm{~Hz}$ ) and normal speech-in-noise perception as revealed by a speech-in-noise test (Lafon 30) from a French language central auditory battery (Demanez et al., 2003) administered at the ENT department of the CUB Hôpital Erasme. All of them were right-handed according to Edinburgh Handedness Inventory (Oldfield, 1971).

This study was approved by the Ethics Committee of the CUB Hôpital Erasme (Brussels, Belgium) and participants signed a written informed consent before participation. They did not receive any financial compensation.

\subsection{Experimental conditions}

Participants underwent 5 experimental conditions lasting 5 min each that were adapted from Vander Ghinst et al. (2016). In the first, task-free, condition they were asked to remain still and to stare at a cross on the wall. The order of the four subsequent conditions was randomized separately for each participant. The task was to listen carefully to a speech stream, henceforth the attended speech, either without noise (noiseless), or mixed with a 
cocktail-party noise at SNR of $+5,0$, and $-5 \mathrm{~dB}$. For each condition, the attended speech was drawn randomly from a set of 6 recordings of different individuals ( 3 males and 3 females) reading a different French text aloud. In the present report, we analysed only the noiseless condition and the noise condition at SNR of $0 \mathrm{~dB}$. The text-to-condition assignment was random and different for all subjects. The noise background consisted of a mix of 6 French speakers' voices (3 women). This type of noise introduces both energetic and informational masking (Hoen et al., 2007; Simpson and Cooke, 2005). The global sound stream (the mix of the attended speech and the background noise) was always displayed at $\sim 60 \mathrm{~dB}$ using a MEG-compatible $60 \times 60 \mathrm{~cm}^{2}$ high-quality flat panel loudspeaker (Panphonics SSH sound shower, Panphonics) facing the subjects, $\sim 2.7 \mathrm{~m}$ away. At the end of each speech stream listening condition, participants were asked 16 questions about the story they had to attend to. They were also asked to rate the intelligibility level of the attended stream on a scale from 0 (totally unintelligible) to 10 (perfectly intelligible).

\subsection{Data acquisition}

During the experimental conditions, participants' brain activity was recorded simultaneously with MEG and EEG at the CUB Hôpital Erasme. Neuromagnetic signals were recorded with a whole-scalp-covering MEG system (Triux, Elekta). The sensor array comprised 306 sensors arranged in 102 triplets of one magnetometer and two orthogonal planar gradiometers. Magnetometers measure the radial component of the magnetic field, while planar gradiometers measure its spatial derivative in the tangential directions. Scalp electric potentials were recorded with a MEG-compatible whole-head and partial face covering low-profile EEG net (256 electrodes MicroCel Geodesic sensor net, Net Amp GES 400 series with MEG compatibility, Electrical Geodesic Inc., Eugene, USA). Electrode 
impedance was kept below $50 \mathrm{k} \Omega$ by means of an electrolyte gel (Redux Gel, Parker Laboratories Inc., New Jersey, USA), and the reference was at Cz. The recordings were performed in a lightweight magnetically shielded room (Maxshield, Elekta), the characteristics of which being described elsewhere (Carrette et al., 2011b; De Tiège et al., 2008). MEG signals were band-pass filtered at $0.1-330 \mathrm{~Hz}$ and sampled at $1000 \mathrm{~Hz}$. EEG signals were low-pass filtered at $450 \mathrm{~Hz}$ and sampled at $1000 \mathrm{~Hz}$. Note that the absence of online high-pass filter for EEG signals did not cause saturation artifacts.

We used 4 head-position indicator coils to monitor subjects' head position during the experimentation. Before the MEG session, and before placing the EEG electrodes, we digitized the location of these coils and at least 300 head-surface points (on scalp, nose, and face) with respect to anatomical fiducials with an electromagnetic tracker (Fastrack, Polhemus). At the end of the experimentation, we digitized the head surface again, along with the location of the $256 \mathrm{EEG}$ electrodes and the $\mathrm{Cz}$ reference with respect to anatomical fiducials.

Finally, subjects' high-resolution 3D-T1 cerebral images were acquired with a magnetic resonance imaging (MRI) scanner (MRI 1.5T, Intera, Philips).

\subsection{Data pre-processing}

Continuous MEG data were first preprocessed off-line using the temporal signal space separation method implemented in MaxFilter software (MaxFilter, Neuromag, Elekta; correlation limit 0.9 , segment length $20 \mathrm{~s}$ ) to suppress external interferences and to correct for head movements (Taulu et al., 2005; Taulu and Simola, 2006). EEG signals were re-referenced to a common average. EEG signals at electrodes affected by excessive noise level (mainly due to bad electrode-skin contact) were reconstructed by interpolation of the 
signals from the surrounding electrodes as done in Perrin et al. (1989). Electrodes were considered noisy when they matched at least one of the three criteria previously described by Bigdely-Shamlo et al. (2015). These three criteria are 1) too high wide-band amplitude, 2) too high ratio between high and low frequency amplitudes, and 3) too low correlation with other channels. The details of the methods and default values used for these three criteria can be found in (Bigdely-Shamlo et al., 2015). Across subjects and conditions, $21.9 \pm 11.8$ (mean \pm SD) electrodes signals were interpolated.

To further suppress physiological artifacts from MEG and EEG data separately, 30 independent components were evaluated from the data band-pass filtered at $0.1-25 \mathrm{~Hz}$ and reduced to a rank of 30 with principal component analysis. Independent components corresponding to heartbeat, eye-blink, and eye-movement artifacts were identified, and corresponding MEG or EEG signals reconstructed by means of the mixing matrix were subtracted from the full-rank data. Across subjects and conditions, the number of components rejected was $2.3 \pm 1.0$ (MEG; mean $\pm \mathrm{SD}$ ) and $6.6 \pm 2.1$ (EEG). Finally, time points at timings $1 \mathrm{~s}$ around remaining artifacts were set to bad. Data were considered contaminated by artifacts when MEG amplitude exceeded $5 \mathrm{pT}$ in at least one magnetometer or $1 \mathrm{pT} / \mathrm{cm}$ in at least one gradiometer, or when EEG amplitude in at least one channel exceeded 10 times its standard deviation. A different rejection scheme was used for MEG and EEG signals because EEG signals tended to be more variable across subjects. It is noteworthy that because the definition of bad data points was common to MEG and EEG signals, analyses of both modalities were carried out on the exact same artefact-free time intervals.

We finally extracted the temporal envelope from the different audio signals (attended speech and global sound). To that aim, audio signals were rectified and low-pass filtered at 
$50 \mathrm{~Hz}$. We did not explore superior methods for extracting the speech envelope (Biesmans et al., 2017), but this is unlikely to affect the results as the resulting envelopes are very similar.

\subsection{Speech brain tracking assessed channel-by-channel}

For each condition, we calculated the coherence-based speech brain tracking as the coherence between the envelope of each audio signal and each MEG and EEG signal. To that aim, time-locked MEG, EEG and envelope of audio signals were segmented into 2000-ms epochs with 1600-ms overlap, affording a $0.5-\mathrm{Hz}$ frequency resolution (Bortel and Sovka, 2014). Epochs contaminated by artifacts were discarded from the analysis, leaving $N_{\mathrm{ep}}=677$ \pm 42 (mean $\pm \mathrm{SD}$ ) epochs in the noiseless condition and $N_{\mathrm{ep}}=689 \pm 35$ epochs in the noise condition. These values did not differ significantly between conditions $\left(t_{9}=-1.18, p=0.27\right.$, paired $t$-test). Coherence was evaluated only for frequencies below $20 \mathrm{~Hz}$, which are critical for speech comprehension (Rosen, 1992), following the formulation of Halliday et al (1995). Furthermore, we specifically analysed coherence values in two frequency ranges of interest: delta frequencies $(0.5 \mathrm{~Hz})$ and theta frequencies (mean coherence across 4-8 Hz). For the sake of comparing coherence values between modalities, we extracted the maximum coherence value across all channels (i.e., all sensors, including both magnetometers and gradiometers for MEG, and all electrodes for EEG). Note that coherence at the frequency bin corresponding to $0.5 \mathrm{~Hz}$ actually reflects coupling in a range of frequencies $f$ around $0.5 \mathrm{~Hz}$, with a sensitivity profile proportional to the Fourier transform of a 2-s-long boxcar function: $\operatorname{sinc}(\pi(f-0.5 \mathrm{~Hz}) / 0.5 \mathrm{~Hz})$. These two frequency intervals have been highlighted in previous studies: the first interval centered on $0.5 \mathrm{~Hz}$ matches with phrasal/sentence rhythmicity (Bourguignon et al., 2013; Gross et al., 2013; Kösem and van Wassenhove, 2016; Molinaro et al., 2016; Vander Ghinst et al., 2016), and, although speech temporal envelope did not 
show a clear peak at 4-8 $\mathrm{Hz}$ (see Figure 1), this frequency interval was specifically analysed here because it has been consistently reported to match with syllable rhythmicity, regardless of the language (Abrams et al., 2008; Ding et al., 2017; Luo and Poeppel, 2007; Molinaro et al., 2016; Peelle et al., 2013; Vander Ghinst et al., 2016; Zion-Golumbic et al., 2012). Of note, in French, the mean syllabic rate is of $\sim 7$ per second (Pellegrino et al., 2011).

For MEG data, a single optimal coherence value was estimated per gradiometer pair as done by Bourguignon et al. (2015). Briefly, the coherence was computed in the optimal direction within the two dimensional space spanned by the two gradiometers. These values of coherence for gradiometer pairs were used for visualization purposes, and for the statistical comparison described in section 2.9 .6 only.

For EEG, coherence was also estimated with current source density distribution estimated via surface Laplacian transformation implemented in Fieldtrip ((Oostenveld et al., 2011; Pernier et al., 1988); http://www.ru.nl/neuroimaging/fieldtrip). Surface Laplacian transformation renders the data reference-free and increases spatial selectivity (Huiskamp, 1990; Oostendorp and van Oosterom, 1996). It was therefore expected to boost coherence values.

For the noiseless condition, all subjects, modalities and frequency ranges of interest separately, we also estimated the minimum amount of data necessary to uncover significant speech brain tracking (see section 2.9.1 for the description of significance assessment). In that analysis, we estimated the maximum coherence across all channels based on the first 1,2,3, $\ldots N_{\text {ep }}$ epochs. We then defined the minimum necessary recording time as the number of epochs for which maximum coherence turns and remains significant, multiplied by $0.4 \mathrm{~s}$ (i.e., epoch length minus epoch overlap). Note that this is an optimistic estimation because it tells how much artifact-free data is needed rather than actual recording time. This is however not a 
problem since the aim was to compare the minimum necessary recording time between modalities.

\subsection{Speech brain tracking assessed globally}

For the noiseless condition, a global value of speech brain tracking was also evaluated for all MEG signals (gradiometers only) at once, for all EEG signals at once, and for all MEG and EEG signals at once (later referred to as MEEG signals). This analysis was motivated by that EEG electrodes are generally sensitive to larger brain volumes than MEG sensors (Hari and Puce, 2017). Consequently, when measuring focal cortical activity, related EEG signals at a given electrode contain more interferences from neighboring brain areas than MEG signals at a given sensor. One could therefore hypothesise that speech brain tracking estimated based on EEG signals would benefit more from a global estimation procedure — which would, e.g., subtract interferences - than that estimated based on MEG signals.

Using the mTRF toolbox (Crosse et al., 2016), we trained a decoder on electrophysiological data (MEG, EEG or MEEG) to reconstruct speech temporal envelope, and estimated its Pearson correlation with real speech temporal envelope. This correlation is often referred to as the reconstruction accuracy (RA), and it provides a global measure of speech brain tracking. A similar approach has been used in previous studies on speech brain tracking (Ding and Simon, 2012; Lalor and Foxe, 2010; O'Sullivan et al., 2014; Zion-Golumbic et al., 2013).

In practice, electrophysiological data were band-pass filtered at $0.2-1.5 \mathrm{~Hz}$ (delta) or 2-8 $\mathrm{Hz}$ (theta), resampled to $10 \mathrm{~Hz}$ (delta) or $40 \mathrm{~Hz}$ (theta) and standardized. A decoder was built based on electrophysiological data from $-500 \mathrm{~ms}$ to $1000 \mathrm{~ms}$ (delta) or from $0 \mathrm{~ms}$ to 250 
ms (theta) with respect to speech temporal envelope. Filtering and delay ranges were as in previous studies for theta frequencies (Ding and Simon, 2012; Lalor and Foxe, 2010; O’Sullivan et al., 2014; Zion-Golumbic et al., 2013), and defined based on an inspection of the temporal response functions for delta frequencies (data not shown). Regularization was applied to limit the norm of the derivative of the reconstructed speech temporal envelope (Crosse et al., 2016), by estimating the decoder for a fixed set of ridge values $\left(\lambda=2^{10}, 2^{12}, 2^{14}\right.$, $\left.2^{16}, 2^{18}, 2^{20}\right)$. In a classical 10 -fold cross-validation approach, the data is split in 10 segments of equal length, the decoder is estimated for 9 segments and tested on the remaining segment, and this procedure is repeated 10 times until all segments have served as test segment. The ridge value yielding the maximum mean reconstruction accuracy is then retained. Due to the maximization procedure, ensuing RAs are inflated. To eliminate this bias, we adopted a 10-fold nested cross-validation scheme (Varoquaux et al., 2017). Following that scheme, for each segment, a 9-fold cross validation was conducted on the remaining 9 segments to select the ridge value that maximizes the RA, and the corresponding decoder was used to estimate the RA for that segment. This procedure led to 10 values of reconstruction accuracy (one for each segment) per subject, frequencies of interest, and audio signal. We refer to the mean value of the RA as the RA-based speech brain tracking value.

\subsection{Coherence analysis in source space}

Individual MRIs were first segmented using the FreeSurfer software (Reuter et al., 2012). Then, MEG and EEG coordinate systems were coregistered to individual subjects' MRI with the MEG-MRI integration software (MRIlab, Neuromag, Elekta), using the three anatomical fiducial points for initial estimation and the head-surface points to manually refine the surface coregistration. The forward models were next computed within a 5-mm grid 
source space for 3 orthogonal source orientations per grid point with MNE suite (Gramfort et al., 2014). Forward modelling was based on a 1-layer boundary element model for MEG data and on a 3-layer one for EEG data. Based on these forward models, we computed a Minimum-Norm-Estimates inverse solution (Dale and Sereno, 1993), with the regularization parameter fixed assuming a SNR of 1 (Hämäläinen et al., 2010). We then used this inverse solution to produce coherence maps in the source space for each condition (noiseless and noise), participant, audio signal (attended and global), frequency band of interest (delta and theta), and recording modality (MEG and EEG). Of notice, the coherence value at each source location was optimized across the three source orientations in a way similar to what was done for the MEG gradiometers. For MEG source reconstruction, both planar gradiometers and magnetometers were used for inverse modeling after dividing each sensor signal (and the corresponding forward-model coefficients) by its noise standard deviation. The noise variance was estimated from the continuous task-free MEG data band-passed through 1-195 Hz, for each sensor separately.

\subsection{Group-level analysis}

A non-linear transformation from individual MRIs to the MNI brain ("Montreal Neurological Institute") was first computed using the spatial normalization algorithm implemented in Statistical Parametric Mapping (SPM8; (Ashburner et al., 1997; Ashburner and Friston, 1999) and then applied to the coherence maps. The resulting maps were then averaged across subjects to produce group-level maps.

We identified the coordinates of the local maxima in group-level coherence maps. Local coherence maxima are sets of contiguous voxels displaying higher coherence value than all other neighbouring voxels (Bourguignon et al., 2012). We only report on local-and 
statistically significant (see below) — coherence maxima and disregard the extent of the clusters of significant coherence. Indeed, cluster extent is hardly interpretable in view of the inherent smoothness of MEG/EEG source reconstruction (Bourguignon et al., 2017; Hämäläinen and Ilmoniemi, 1994; Hari et al., 1988; Sekihara et al., 2005; Wens et al., 2015) .

In one subject, probably due to head movements during the MRI acquisition, the inner and outer skull layers did intersect, leading to a failure to compute the EEG forward model. For this reason, we were able to perform the source-level analysis on 9 participants only.

\subsection{Statistical analyses}

\subsubsection{Coherence-based speech brain tracking}

For each listening condition, frequency band of interest, and recording modality, the statistical significance of individual coherence values was assessed with maximum-based statistics derived from surrogate data, which takes into account both the multiple comparisons across channels and the temporal autocorrelation within signals. For each subject, 1000 surrogate coherence distributions across channels were generated, as was done for genuine coherence values but with the audio signals replaced by their Fourier-transform surrogates, which preserves their power spectrum by replacing the phase of their Fourier coefficients by random numbers in the range $[-\pi ; \pi]$ (Faes et al., 2004). Then, the maximum coherence value across all channels was extracted for each surrogate simulation. Finally, the $95^{\text {th }}$ percentile of this maximum coherence value yielded the coherence threshold at $p<0.05$. Across subjects and conditions, the coherence thresholds were $0.0436 \pm 0.0028$ (mean $\pm \mathrm{SD}$; MEG, delta), $0.0375 \pm 0.0030$ (EEG, delta), $0.0126 \pm 0.0006$ (MEG, theta), and $0.0112 \pm$ 0.0006 (EEG, theta). 


\subsubsection{Reconstruction accuracy}

The significance of individual RA values was assessed with one sample t-tests across the 10 validation runs.

\subsubsection{Comparison of speech brain tracking values}

Maximum coherence-based speech brain tracking values across all channels, RA-based speech brain tracking values and minimum necessary recording times were compared between recording modalities (MEG vs. EEG; MEG vs. MEEG; EEG vs. MEEG) with paired $t$-tests. The same approach was also used to compare maximum coherence-based speech brain tracking values obtained based on EEG with versus without surface Laplacian transformation.

A link between RA values obtained based on MEG and EEG signals was sought for with Spearman correlation.

\subsubsection{Effect of noise on the intelligibility of the attended speech}

We used paired, two-tailed $t$-tests to determine the effect of the SNR (noiseless, noise) on intelligibility ratings and on scores on the comprehension questions.

2.9.5 Comparison of speech brain tracking with the attended speech vs. global sound in the noise condition

We evaluated whether subjects brain signals were specifically tracking the attended speech or the global sound. To that aim, we estimated for each subject the tune-in index as $\left(C o h_{\text {attended }}-C o h_{\text {global }}\right) /\left(C o h_{\text {attended }}+C o h_{\text {global }}\right)$, where $C o h_{\text {attended }}$ (respectively $\left.C o h_{\text {global }}\right)$ is the maximum across all channels of coherence-based speech brain tracking values estimated with 
the attended (respectively global) speech signal. Tune-in indices were compared to 0 with one sample, two-tailed $t$-tests, and were compared between modalities with paired, two tailed $t$-tests.

\subsubsection{Effect of the noise on coherence levels and hemispheric lateralization}

To determine the effect of the auditory noise on speech brain tracking magnitude and hemispheric lateralization, coherence-based speech brain tracking values were assessed with repeated-measures ANOVA. In that analysis, coherence-based speech brain tracking was taken as the maximum coherence value across the 9 gradiometer pairs (MEG) or 20 electrodes (EEG) of maximum group-averaged coherence in the left and right hemispheres separately, and at delta and theta frequencies separately. Note that here, we used the MEG coherence values optimized across orientations within each gradiometer pair (see section 2.5).

\subsubsection{Coherence in source space}

The statistical significance of the local coherence maxima observed in group-level source coherence maps was assessed with a nonparametric maximum-based permutation test

(Nichols and Holmes, 2001). The following procedure was performed for each listening condition, frequency band of interest, recording modality, and brain hemisphere separately. First, subject- and group-level task-free coherence maps were computed, as done for the different listening conditions, but with MEG signals replaced by task-free MEG signals and sound signals unchanged. Group-level difference maps were then obtained by subtracting listening condition coherence maps and task-free coherence maps. Under the null hypothesis that coherence maps are the same whatever the experimental condition, the labelling "listening condition" and "task-free" are exchangeable at the subject-level before group-level 
difference map computation (Nichols and Holmes, 2001). To reject this hypothesis and to compute a threshold of statistical significance for the correctly labelled difference map, the permutation distribution of the maximum of the difference map's absolute value was computed from all possible 512 permutations. The threshold at $p<0.05$ (intrinsically corrected for the multiple comparison issue across sources) was computed as the $95^{\text {th }}$ percentile of the permutation distribution (Nichols and Holmes, 2001). All supra-threshold local coherence maxima were interpreted as indicative of brain regions showing statistically significant coupling with the audio signals.

\subsubsection{Confidence on peak coherence coordinates and comparison between MEG and EEG.}

The coordinates of the local maxima identified in MEG and EEG coherence maps were statistically compared using the location-comparison approach proposed by (Bourguignon et al., 2017). That method uses a bootstrap procedure (Efron, 1979) to estimate the sample distribution of coordinates of local coherence maxima in MEG and EEG maps and tests the null hypothesis that the distance between them is zero. Briefly, we generated 1000 group-level MEG and EEG maps by random bootstrapping from the individual maps, and identified the coordinates of the local maxima closest to the genuine maxima location. The resulting sample distribution of coordinates difference was then submitted to a multivariate location test evaluating the probability that this difference is zero (Bourguignon et al., 2017). That test tightly relates to the multivariate $T^{2}$ test (Hotelling, 1931) and assumes that the sample distribution of coordinates difference is normal.

In that framework, the $95 \%$ confidence volume $V$ and surface $S$ were also estimated for each local maximum. Specifically, $V$ was computed as the volume of an ellipsoid that contains $95 \%$ of the sample coordinates of a given local maximum, and $S$, as the surface of 
an ellipse that contains $95 \%$ of the sample coordinates projected orthogonally onto the plane of maximum variance. Assuming the sample coordinates are normally distributed, we have $V=k_{V}^{3 / 2} \frac{4}{3} \pi \sigma_{1} \sigma_{2} \sigma_{3}$ and $S=k_{S} \pi \sigma_{1} \sigma_{2}$, where $\sigma_{i}^{2}(i \in\{1,2,3\})$ are the eigenvalues of sample coordinates covariance matrix sorted in ascending order, and $k_{V}$ and $k_{S}$ are the 95-percentile of the $\chi_{3}^{2}$ and $\chi_{2}^{2}$ distributions respectively.

\section{Results}

\subsection{Coherence-based speech brain tracking values in the noiseless condition}

\section{INSERT TABLE 1 ABOUT HERE} INSERT FIGURE 2 ABOUT HERE

Figure 2 presents speech brain tracking values obtained in the noiseless condition with the coherence-based approach, based on MEG $\left(\mathrm{Coh}_{\mathrm{MEG}}\right)$ and EEG recordings $\left(\mathrm{Coh}_{\mathrm{EEG}}\right)$. As previously reported in the literature, coherence at $0.5 \mathrm{~Hz}$ (delta frequencies) and $4-8 \mathrm{~Hz}$ (theta frequencies) was maximum over temporo-frontal brain areas. The topographic distribution of group-level coherence with EEG signals suggests that the underlying sources are mainly radial at delta frequencies, and tangential at theta frequencies. However, individual coherence maps, which are not presented here, demonstrated a large inter-individual variability. 
Table 1 and Figure 3 present the maximum $\mathrm{Coh}_{\mathrm{MEG}}$ and $\mathrm{Coh}_{\mathrm{EEG}}$ values across all channels. At delta frequencies, all subjects displayed significant $\mathrm{Coh}_{\mathrm{MEG}}$ and 8/10 displayed significant $\mathrm{Coh}_{\mathrm{EEG}}(p s<0.05)$. At theta frequencies, $\mathrm{Coh}_{\mathrm{MEG}}$ was significant in $8 / 10$ subjects and $\mathrm{Coh}_{\mathrm{EEG}}$ in $6 / 10$ subjects. Importantly, maximum $\mathrm{Coh}_{\mathrm{MEG}}$ was significantly higher than maximum $\mathrm{Coh}_{\mathrm{EEG}}$ by a factor of 3.23 on average at delta frequencies $\left(t_{9}=7.25, p<0.001\right)$ and of 1.85 at theta frequencies $\left(t_{9}=3.55, p=0.0062\right.$; see Table 1$)$.

Applying the surface Laplacian transformation to EEG signals further decreased coherence-based speech brain tracking values. This effect was statistically significant at delta frequencies $\left(t_{9}=2.65, p=0.027\right)$ but not at theta frequencies $\left(t_{9}=1.52, p=0.16\right)$. Therefore, in what comes next, we only report on results obtained based on non-transformed mean-referenced EEG signals.

\subsection{Minimum necessary recording time in the noiseless condition}

INSERT FIGURE 4 ABOUT HERE

Figure 4 presents the evolution of $\mathrm{Coh}_{\mathrm{MEG}}$ and $\mathrm{Coh}_{\mathrm{EEG}}$ with recording length in the noiseless condition at delta and theta frequencies, illustrating that differences in coherence levels directly translated into differences in minimum necessary recording time. In the 8 subjects with significant $\mathrm{Coh}_{\mathrm{MEG}}$ and $\mathrm{Coh}_{\mathrm{EEG}}$ at delta frequencies, the minimum necessary recording time was 3.68 times longer in EEG (127.2 $\pm 67.9 \mathrm{~s})$ than in MEG (34.6 $\pm 20.7 \mathrm{~s})$ and this difference was statistically significant $\left(t_{7}=3.43, p=0.011\right)$. In the two subjects lacking significant $\mathrm{Coh}_{\mathrm{EEG}}$, the minimum necessary MEG recording time was $9.2 \mathrm{~s}$ and $230 \mathrm{~s}$. In the six subjects with significant $\mathrm{Coh}_{\mathrm{MEG}}$ and $\mathrm{Coh}_{\mathrm{EEG}}$ at theta frequencies, the minimum necessary recording time was 1.86 times longer in EEG $(195.3 \pm 59.0 \mathrm{~s})$ than in MEG (105.3 $\pm 63.5 \mathrm{~s})$ and this difference was statistically significant $\left(t_{5}=2.63, p=0.047\right)$. In the four 
remaining subjects, two lacked significant $\mathrm{Coh}_{\mathrm{MEG}}$ and $\mathrm{Coh}_{\mathrm{EEG}}$, and two lacked significant $\mathrm{Coh}_{\mathrm{EEG}}$ while having significant $\mathrm{Coh}_{\mathrm{MEG}}$ uncovered in a minimum necessary recording time of $129.6 \mathrm{~s}$ and $130.0 \mathrm{~s}$.

\subsection{RA-based speech brain tracking values in the noiseless condition}

INSERT FIGURE 5 ABOUT HERE

Table 1 and Figure 5 present speech brain tracking values obtained in the noiseless condition with the RA-based approach. With this approach, a single value of RA was obtained for all MEG sensors $\left(\mathrm{RA}_{\mathrm{MEG}}\right)$, for all EEG electrodes $\left(\mathrm{RA}_{\mathrm{EEG}}\right)$, and for all MEG and EEG channels combined optimally $\left(\mathrm{RA}_{\mathrm{MEEG}}\right)$.

The ridge parameter selected through nested cross validation was well within the explored range (from $2^{10}$ to $2^{20}$ ). Indeed, with the selected ridge value written $2^{l}$, the mean $l$ across validation runs was $14.3 \pm 1.5$ (MEG, mean $\pm \mathrm{SD}$ across subjects), $13.9 \pm 2.6$ (EEG) and $14.9 \pm 1.4(\mathrm{MEEG})$ at delta frequencies and $15.0 \pm 2.3(\mathrm{MEG}), 15.1 \pm 1.3(\mathrm{EEG})$ and $15.4 \pm 1.7(\mathrm{MEEG})$ at theta frequencies. Across all modalities, frequency ranges, subjects and validation runs, extreme values of $l(10$ or 20$)$ were selected in $10.2 \%$ of the instances.

$\mathrm{RA}_{\mathrm{MEG}}, \mathrm{RA}_{\mathrm{EEG}}$ and $\mathrm{RA}_{\mathrm{MEEG}}$ were significant in all subjects and at both frequencies ( $p s$ $<0.05$ ), except for $\mathrm{RA}_{\mathrm{EEG}}$ in one subject at theta frequencies. $\mathrm{RA}_{\mathrm{MEG}}$ was higher than $\mathrm{RA}_{\mathrm{EEG}}$, by factors 1.56 at delta frequencies $\left(t_{9}=5.11, p=0.0006\right)$ and 1.69 at theta frequencies $\left(t_{9}=\right.$ -4.19, $p=0.0024) . \mathrm{RA}_{\mathrm{MEG}}$ and $\mathrm{RA}_{\mathrm{EEG}}$ were strongly correlated, both at delta $(r=0.78, p=$ 0.012 , Spearman correlation) and theta frequencies $(r=0.70, p=0.031) . \mathrm{RA}_{\mathrm{MEG}}$ did not differ significantly from $\mathrm{RA}_{\mathrm{MEEG}}$ (delta frequencies, $t_{9}=1.42, p=0.19$; theta frequencies, $t_{9}=2.02$, 
$p=0.074$ ), while $\mathrm{RA}_{\mathrm{EEG}}$ was significantly lower than $\mathrm{RA}_{\mathrm{MEEG}}$ (delta frequencies, $t_{9}=5.12, p$ $=0.0006$; theta frequencies, $t_{9}=6.18, p=0.0002$ ).

\subsection{Comparison between noiseless and noise conditions in MEG and EEG}

The introduction of the background noise significantly impacted speech comprehension. Indeed, subjects gave higher intelligibility ratings in the noiseless than in the noise condition (mean $\pm \mathrm{SD}, 9.9 \pm 0.3$ vs. $4.4 \pm 2.0 ; t_{9}=8.0, p<0.001$, paired two-tailed $t$-test). Likewise, they answered more accurately to the comprehension questions in the noiseless than in the noise condition $\left(14.1 \pm 1.3\right.$ vs. $\left.11.1 \pm 2.0 ; t_{9}=5.2, p<0.001\right)$.

\section{INSERT FIGURE 6 ABOUT HERE}

Figure 6 presents $\mathrm{Coh}_{\mathrm{MEG}}$ and $\mathrm{Coh}_{\mathrm{EEG}}$ estimated with the attended speech in the noise condition. Coherence topographies were similar to those in the noiseless condition (see Figure 2). Coherence values were overall similar to those obtained in the noiseless condition, except for $\mathrm{Coh}_{\mathrm{MEG}}$ values at delta frequencies that were $\sim 35 \%$ lower. At delta frequencies, $\mathrm{Coh}_{\mathrm{MEG}}$ and $\mathrm{Coh}_{\mathrm{EEG}}$ were significantly stronger when estimated with the attended speech than with the global sound (MEG, mean $\pm \mathrm{SD}$ tune-in index $=0.085 \pm 0.064, t_{9}=4.24, p=$ 0.0022; EEG, tune-in index $\left.=0.084 \pm 0.063, t_{9}=4.26, p=0.0021\right)$. The same effect was observed at theta frequencies with MEG (tune-in index $=0.078 \pm 0.070, t_{9}=3.48, p=$ 0.0069 ) but did not reach statistical significance with EEG (tune-in index $=0.033 \pm 0.052, t_{9}$ $=1.98, p=0.079)$. However, tune-in indices did not differ significantly between modalities (delta, $t_{9}=0.09, p=0.93$; theta, $t_{9}=1.83, p=0.10$ ). 
We formally compared coherence values between conditions with a three-way (2 conditions $\times 2$ hemispheres $\times 2$ modalities) repeated measures ANOVA at delta and theta frequencies separately. In this analysis, the dependent variable was the maximum coherence value across a left and a right clusters of channels.

At delta frequencies, the three-way ANOVA revealed a significant main effect of modality $\left(F_{1,9}=57.84, p<0.0001\right)$, a significant double interaction between condition and modality $\left(F_{1,9}=6.31, p=0.033\right)$, and a significant triple interaction between hemisphere, condition and modality $\left(F_{1,9}=5.90, p=0.038\right)$. The strong effect of modality (confirming that $\mathrm{Coh}_{\mathrm{MEG}}$ is higher than $\mathrm{Coh}_{\mathrm{EEG}}$ ) suggested conducting the ANOVA separately for $\mathrm{Coh}_{\mathrm{MEG}}$ and $\mathrm{Coh}_{\mathrm{EEG}}$. When performed on $\mathrm{Coh}_{\mathrm{MEG}}$ values, the ANOVA revealed a significant interaction between hemisphere and condition $\left(F_{1,9}=5.53, p=0.043\right)$, a significant main effect of condition $\left(F_{1,9}=5.62, p=0.042\right)$, and no main effect of hemisphere $\left(F_{1,9}=0.18, p=\right.$ 0.68). Post-hoc $t$-tests revealed that right hemisphere $\mathrm{Coh}_{\mathrm{MEG}}$ was significantly higher in the noiseless (mean $\pm \mathrm{SD}, 0.176 \pm 0.078)$ than in the noise condition $\left(0.092 \pm 0.056, t_{9}=-2.77, p\right.$ $=0.022$ ) while such difference was not significant in the left hemisphere (noiseless, $0.142 \pm$ 0.068 ; noise, $0.113 \pm 0.043 ; t_{9}=-1.32, p=0.22$ ). Such effect was not found based on EEG recordings as the ANOVA performed on $\mathrm{Coh}_{\mathrm{EEG}}$ values showed no effect of condition $\left(F_{1,9}=\right.$ $0.60, p=0.46)$, hemisphere $\left(F_{1,9}=0.24, p=0.64\right)$ or interaction thereof $\left(F_{1,9}=0.12, p=0.73\right)$.

At theta frequencies, the three-way ANOVA revealed a significant main effect of modality $\left(F_{1,9}=37.11, p=0.0002\right)$ and no other main effects nor interactions $(p s>0.05)$. Again, $\mathrm{Coh}_{\mathrm{MEG}}\left(0.0237 \pm 0.0071\right.$, mean $\pm \mathrm{SD}$ of mean $\mathrm{Coh}_{\mathrm{MEG}}$ across hemispheres and conditions) was higher than $\mathrm{Coh}_{\mathrm{EEG}}(0.0109 \pm 0.0036)$. 


\subsection{Brain sources of speech brain tracking}

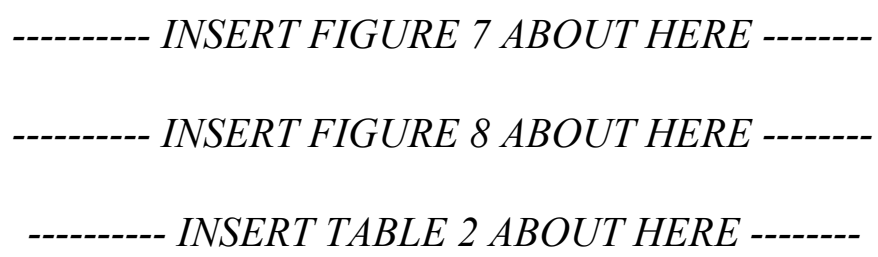

Figures 7 and 8 present the maps of group-level $\mathrm{Coh}_{\mathrm{MEG}}$ and $\mathrm{Coh}_{\mathrm{EEG}}$ at delta and theta frequencies in the noiseless (Figure 7) and in the noise (Figure 8) conditions. Table 2 presents the corresponding peak MNI coordinates, the confidence on these coordinates as well as the result of their comparison between MEG and EEG.

Overall, coherence peaked in bilateral auditory regions in both conditions. All coherence values at these local maxima were significant $(p s<0.05)$ except for left-hemisphere $\mathrm{Coh}_{\mathrm{EEG}}$ at theta frequencies in the noise condition $(p=0.51)$. All local maxima were in the supratemporal auditory cortex except for those of $\mathrm{Coh}_{\mathrm{MEG}}$ at delta frequencies in the noiseless condition that localized in bilateral posterior superior temporal sulcus (see Table 2 for MNI coordinates) and in the left inferior frontal lobule (MNI coordinates $\left.\left[\begin{array}{lll}-63 & 8 & 22\end{array}\right] \mathrm{mm}\right)$. Of notice, the local maximum of left hemisphere $\mathrm{Coh}_{\mathrm{EEG}}$ at theta frequencies in the noiseless condition was substantially more anterior and ventral than other local maxima, in the white matter underlying the frontal pole of the middle temporal gyrus. However, this local maximum barely exceeded the significance threshold and was associated with very large localization uncertainty: its volume of confidence was $\sim 70 \mathrm{~cm}^{3}$. It is therefore still compatible with a localization in supratemporal auditory cortex.

$\mathrm{Coh}_{\mathrm{EEG}}$ peaked generally deeper than $\mathrm{Coh}_{\mathrm{MEG}}$. A formal test comparing the location of peak $\mathrm{Coh}_{\mathrm{EEG}}$ and $\mathrm{Coh}_{\mathrm{MEG}}$ revealed a significant difference in all right-hemisphere peaks, and 
in the left hemisphere peak in the noiseless condition at delta frequencies (see Table 2). Finally, the confidence volume of local maxima of $\mathrm{Coh}_{\mathrm{EEG}}$ was 5-78 times larger than that of $\mathrm{Coh}_{\mathrm{MEG}}$. This large difference can be partly explained by that confidence volumes of peak $\mathrm{Coh}_{\mathrm{MEG}}$ are compressed in the orientation perpendicular to the skull, simply because $\mathrm{Coh}_{\mathrm{MEG}}$ peaked systematically at the surface. This did not happen for deeper-localized $\mathrm{Coh}_{\mathrm{EEG}}$ sources. Hence, an index of dispersion more comparable between the two modalities is the confidence surface. That is, an estimation of the surface in the plane of maximum dispersion containing the dominant source of speech brain tracking with $95 \%$ confidence. Still, the confidence surface of local maxima of $\mathrm{Coh}_{\mathrm{EEG}}$ was 3-12 times larger than those of $\mathrm{Coh}_{\mathrm{MEG}}$. 


\section{Discussion}

This simultaneous MEG-EEG study demonstrates that speech brain tracking values estimated based on MEG recordings are higher than those estimated based on EEG recordings, and that speech brain tracking requires $\sim 3$ times shorter recordings with MEG than with EEG to become significant. As likely consequence of this discrepancy, some previously reported findings related to speech in noise perception were replicated based on MEG but not EEG recordings.

\subsection{Speech brain tracking values estimated based on MEG vs. EEG}

The main objective of this study was to compare the potential of MEG and EEG to uncover significant speech brain tracking. As a result, both modalities yielded substantially different coupling estimates in terms of magnitude and source localization.

In the channel-by-channel analysis, the coupling estimated based on MEG recordings was systematically higher than that estimated based on EEG recordings, by a factor of $\sim 3$ at delta frequencies and $\sim 2$ at theta frequencies for the noiseless condition. Since significance thresholds for coherence estimates decrease asymptotically as the inverse of the amount of data points available (Halliday et al., 1995), it can be inferred that EEG recordings need to be 2 or 3 times longer than MEG recordings to uncover significant speech brain tracking. Empirically supporting this inference, the minimum necessary recording time to uncover significant coherence-based speech tracking was 3.7 (delta) or 1.9 (theta) times longer in EEG than in MEG. This explains why the detection rate of significant speech brain tracking observed here was systematically higher with MEG than with EEG. Hence, in experiments in which several speech brain tracking conditions need to be recorded, MEG should be preferred over EEG to keep experiment duration comfortable for the participants. 
The discrepancy in speech brain tracking values between modalities could have been induced by differences in spatial selectivity they afford. Indeed, EEG electrodes are sensitive to a larger brain volume than MEG sensors, especially so for planar gradiometers (Hämäläinen et al., 1993a). One could therefore hypothesize that speech brain tracking could be seen with equal accuracy based on both modalities, provided that adequate channel combinations be used. Still, surface Laplacian transformation decreased rather than increased EEG-based speech brain tracking values. Also, RA-based speech brain tracking values were still 1.56 (delta) or 1.69 (theta) times higher when estimated based on MEG than EEG recordings. RAs are correlation values and significance thresholds for correlation estimates decrease asymptotically as the inverse of the square root of the amount of data points available. Accordingly, when assessed with all channels at once, EEG recordings still needed to be 2.4 (delta) or 2.9 (theta) times longer than MEG recordings to uncover significant speech brain tracking. In sum, these results do not support the hypothesis that optimal channel combination enables EEG to uncover speech brain tracking with similar accuracy as MEG.

In the framework of RA, we could also evaluate the yield of combining MEG and EEG signals to estimate speech brain tracking. As a result, RA values estimated based on combined MEG and EEG signals were 7\% higher than those estimated based on MEG signals only. This effect was not significant, possibly because of statistical power issues intrinsic to studying small sample size (10 subjects here). Still, we have showed that adding EEG information to that already present in MEG has at best a mild effect on speech brain tracking estimation. In the same line, the result that $\mathrm{RA}_{\mathrm{EEG}}$ correlates strongly with $\mathrm{RA} \mathrm{A}_{\mathrm{MEG}}$ suggests that, at least for speech brain tracking investigations, both MEG and EEG recorded 
similar-rather than complementary-information, though MEG does so with a more advantageous SNR.

The difference between speech brain tracking values estimated based on MEG and EEG recordings probably pertains to that SNR is higher in MEG than in EEG recordings. Such SNR difference between MEG and EEG modalities has been previously reported for the superficial portion of the cortex, leading to the conclusion that MEG is more suited than EEG to record superficial and focal sources (Goldenholz et al., 2009). This SNR difference is at least partly explained by that the scalp distribution of electric potential is more diffuse than the corresponding pattern of the radial component of the magnetic field (Hari and Puce, 2017). EEG scalp patterns are diffuse because secondary currents spread laterally at the interfaces between the different mediums of the head (cerebrospinal fluid, skull and scalp tissues) since those have strikingly distinct conductivities. In contrast, magnetic fields are essentially unaffected by these different layers because they all have a magnetic permeability close to that of the vacuum. Consequently, magnetic field spread depends only on the distance between brain sources and recording site (Hari and Puce, 2017). Another source of SNR difference between MEG and EEG signals lays in that MEG signals arise predominantly from close-to-the-scalp tangential currents, while EEG signals also receive contribution from radial and deeper sources (Ahlfors et al., 2010; Hari and Puce, 2017). Consequently, signals related to neuronal activity close to the scalp can be contaminated by a larger amount of interfering random neuronal activity when recorded by EEG.

Speech brain tracking sources estimated based on EEG recordings were systematically deeper than those estimated based on MEG recordings. The origin of this difference is unclear. It may relate to inaccuracies in EEG source reconstruction due to errors in forward model estimation (Hämäläinen et al., 1993b). That is because electric currents are heavily 
distorted by the skull in a way that is difficult to model (Hämäläinen and Hari, 2002). In line with that interpretation, it has been reported that in presence of anisotropic layers (like the bone), superficial sources in EEG may be localized deeper and weaker than with MEG (Wolters et al., 2006). Alternatively, EEG signals may have received a greater contribution from deeper sources of speech brain tracking that are not seen by MEG.

\subsection{Comparison between $M E G$ and EEG in other studies}

Even though MEG and EEG are widely known to capture different neuronal activity, surprisingly very few studies have quantified this discrepancy in sensory or cognitive processes. Most of the studies comparing EEG and MEG are clinical studies investigating the yield of both modalities for the identification and localization of interictal epileptiform discharges in epilepsy patients (Iwasaki et al., 2005; Knake et al., 2006; Paulini et al., 2007; Wheless et al., 1999). These studies have demonstrated that MEG captures epileptiform discharges in $\sim 70 \%$ of the patients, a few percent of whom have normal EEG (De Tiège et al., 2012; Knowlton, 2008, 2006; Stefan et al., 2011). In patients with clinical suspicion of epilepsy but with normal EEG, MEG has $\sim 20 \%$ of sensitivity (Duez et al., 2016). These studies support the claim that MEG and EEG have different sensitivity profiles to different brain activities, but they do not quantify per se discrepancy in signal magnitude, simply because epilepsy is a too heterogeneous brain disorder in terms of source location and epileptic activity.

In a study aiming at assessing the test-retest reliability of $40-\mathrm{Hz}$ auditory steady-state responses, authors quantified with both MEG and EEG the inter-trial coherence (ITC) value induced by auditory white noise and click trains (Legget et al., 2017). Although exact ITC values were not reported in the text, it can be inferred from their figure 3 that group-level ITC 
estimated based on MEG recordings was $\sim 2$ times higher than that estimated based on EEG recordings (Legget et al., 2017). ITC is a measure of phase synchronization with respect to a given stimulation onset in the time-frequency domain (Legget et al., 2017; Tallon-Baudry et al., 1996). It is easy to show that, in the presence of noise only, ITC decreases as the square root of the number of available data segments. Hence, we can conclude that $40-\mathrm{Hz}$ auditory steady state responses can be recovered with MEG based on $\sim 4$ times shorter recordings than required from EEG. This order of magnitude is close to that hereby reported for speech brain tracking.

In the visual domain, steady-state visual-evoked responses at theta (4-8 Hz) and upper alpha (10-14 Hz) frequencies are seen with both MEG and EEG (Thorpe et al., 2007). Again, inspection of values provided in figures but not in the text suggests that SNR in MEG responses is at least 2 times higher than that in EEG responses. However, responses at low-beta frequencies (15-20 Hz) were observed with EEG but not with MEG (Thorpe et al., 2007).

Finally, some perceptual studies have shown that MEG and EEG do not uncover the same brain processes with identical accuracy. For example, the amplitude of the P2 auditory evoked response is modulated by the acoustic complexity in a way that is more prominent in EEG than in MEG (Shahin et al., 2007). This discrepancy likely pertains to that sources of the P2 component are close to radial (Shahin et al., 2007). It also reminds us that our result that EEG recordings need to be 3 times longer than MEG recordings to uncover significant effects is restricted to the specific case of speech brain tracking, i.e., the tracking by brain signals of speech temporal envelope at delta and theta frequencies.

This brief review highlights the importance of quantifying the discrepancy between MEG and EEG recordings for each functional process separately. Depending on source 
location and orientation, MEG and EEG may not afford the same statistical power and see the same effects.

\subsection{Effect of added auditory noise on speech brain tracking}

A secondary objective of this study was to compare the potential of MEG and EEG to uncover previously reported relevant effects related to speech brain tracking in noisy conditions (Vander Ghinst et al., 2016). Three previously reported findings related to speech perception in noise were replicated based on MEG but not EEG recordings: 1) speech brain tracking at theta frequencies is significantly stronger with the attended speech than with the global auditory scene, 2) speech brain tracking at delta frequencies dampens when noise is added, and 3) this dampening is stronger in the right (vs. left) hemisphere. The only effect reproduced by both MEG and EEG was that speech brain tracking at delta frequencies is significantly stronger with the attended speech than with the global auditory scene. This discrepancy in results may be explained by the SNR difference in recordings of the two modalities, in line with our result that $\sim 3$ times less MEG than EEG data is needed to uncover speech brain tracking. Alternatively, it could reflect a difference in sensitivity to tangential vs. radial neocortical sources.

The stronger dampening of delta speech brain tracking in the right (vs. left) hemisphere might have been seen with MEG only simply because responses from both hemispheres are better segregated spatially in MEG than EEG. Undermining this explanation, EEG scalp distribution of coherence at delta frequencies was maximal over bilateral temporal electrodes. Poor spatial segregation in EEG is typically seen for auditory evoked cortical responses such as N1 and P2 components (Pereira et al., 2014). It is also the case of tracking at theta frequencies where EEG scalp distribution of coherence maximum at the vertex and at 
bilateral temporo-occipital electrodes is compatible with N1 topography, as also evident in previous studies (Müller et al., 2018; Ng et al., 2013).

At theta frequencies both MEG and EEG did not reveal a significant difference between noiseless and noise conditions. This finding is well in line with previous results by Vander Ghinst et al. (2016) and Ding and Simon (2013). Vander Ghinst et al. (2016) found that the brain tracks the attended speech at theta frequencies with similar strength in the absence of noise and when it is mixed with a cocktail party noise at $0 \mathrm{~dB}$. Speech brain tracking was however compromised at lower SNR $(-5 \mathrm{~dB}$ and $-10 \mathrm{~dB})$. Ding and Simon (2013) reported that speech brain tracking at theta frequencies remains stable as long as speech is intelligible. Alghouth the speech SNR we used in the present study $(0 \mathrm{~dB})$ did lower speech comprehension and intelligibility ratings, it essentially left speech intelligible. As the absence of significant difference between noiseless and noise conditions is in essence a negative effect, an interaction between modalities and conditions was not expected and not found, despite there being a significant effect of modality confirming that the level of theta speech brain tracking is higher with MEG than EEG.

Finally, speech brain tracking at theta frequencies assessed based on MEG but not EEG recordings was significantly stronger when estimated with the attended speech than with the global sound. This suggests that MEG outperformed EEG at the task of detecting selective speech tracking in noise. However, a direct comparison of tune-in indices between MEG and EEG did not support this conclusion.

\subsection{Limitations and practical considerations}

In the present study, EEG signals were recorded with MEG-compatible passive EEG electrodes. Passive systems provide arguably lower SNR than active systems, wherein signals 
are amplified on the scalp to minimize interferences picked up by the cables. This advantage of active over passive electrodes is however only mildly supported by empirical studies on the topic (Cencen et al., 2016; Laszlo et al., 2014). Furthermore, the impact of ambient noise was certainly mitigated in our setting since EEG signals were recorded in a shielded room. Supporting that assumption, a previous study using 128 active EEG electrodes to estimate RA with an approach highly similar to ours found mean RA values across their group of 0.054 (O'Sullivan et al., 2014), which is rather close to our mean value of 0.0615. Still, further studies are needed to generalize our results to other types of EEG systems.

The present study was conducted on a small sample of subjects $(n=10)$. This limited sample size was likely the reason why we could not evidence the benefit of combining MEG with EEG (over MEG alone) to estimate speech brain tracking, and why selective tracking of the attended speech (vs. global sound) at theta frequencies came significant based on MEG but not EEG recordings, despite the fact that such selective tracking was not significantly better seen with MEG than EEG. Nevertheless, our restricted sample was enough to reliably observe that speech brain tracking is better seen with MEG than EEG, an effect seen in all subjects and frequencies, except for one subject at theta frequencies.

We have demonstrated that MEG largely outperforms EEG in estimating speech brain tracking, i.e., the coupling between cortical activity and heard speech. However, various practical aspects of currently existing MEG systems preclude their large-scale use. MEG recordings may prove challenging in participants wearing cochlear implant, teeth braces or any metallic devices, because all these generate strong interferences. Mitigating this limitation, most of these nearby interferences can be substantially subtracted from the MEG signals of interest with appropriate preprocessing (Bourguignon et al., 2016; Carrette et al., 2011a; Hillebrand et al., 2013; Mäkelä et al., 2007; Medvedovsky et al., 2009; Taulu and 
Hari, 2009; Taulu and Simola, 2006). As another limitation, MEG helmets do not adapt to participants' head size, which implies that individuals with exceptionally large head do not fit in the helmet, and more importantly, when assessing children, the large sensor-brain distance may potentially diminish the SNR (Wehner et al., 2008). And the most important limitations is undoubtedly the high cost related to the MEG system itself, maintenance, helium consumption, and the shielding room required to isolate the brain signals from external magnetic fields (Baillet, 2017). These costs are a barrier to large-scale use of MEG. In comparison, recording with EEG is rather cheap, even with high-density systems. The situation for MEG might however change drastically in a near future. Indeed, a novel generation of MEG sensor types is currently being developed that does not require being immersed in liquid helium (Boto et al., 2018). Shortly, these optically-pumped magnetometers could enable the measurement of MEG signals on-scalp with reasonable SNR, using a portable cap as currently done in EEG (Knappe et al., 2014).

\subsection{Conclusion}

We have demonstrated that EEG recordings need to be $\sim 3$ times longer than MEG recordings to uncover significant speech brain tracking. This result should help researchers and clinicians design their recording procedure to evaluate speech brain tracking.

\section{References}

Abrams, D.A., Nicol, T., Zecker, S., Kraus, N., 2008. Right-hemisphere auditory cortex is dominant for coding syllable patterns in speech. J. Neurosci. 28, 3958-3965.

Ahissar, E., Nagarajan, S., Ahissar, M., Protopapas, A., Mahncke, H., Merzenich, M.M., 2001. Speech comprehension is correlated with temporal response patterns recorded from auditory cortex. Proceedings of the National Academy of Sciences 98, 13367-13372.

Ahlfors, S.P., Han, J., Belliveau, J.W., Hämäläinen, M.S., 2010. Sensitivity of MEG and EEG to source orientation. Brain Topogr. 23, 227-232. 
Ashburner, J., Friston, K.J., 1999. Nonlinear spatial normalization using basis functions. Hum. Brain Mapp. 7, 254-266.

Ashburner, J., Neelin, P., Collins, D.L., Evans, A., Friston, K., 1997. Incorporating prior knowledge into image registration. Neuroimage 6, 344-352.

Biesmans, W., Das, N., Francart, T., Bertrand, A., 2017. Auditory-Inspired Speech Envelope Extraction Methods for Improved EEG-Based Auditory Attention Detection in a Cocktail Party Scenario. IEEE Trans. Neural Syst. Rehabil. Eng. 25, 402-412.

Bigdely-Shamlo, N., Mullen, T., Kothe, C., Su, K.-M., Robbins, K.A., 2015. The PREP pipeline: standardized preprocessing for large-scale EEG analysis. Front. Neuroinform. $9,16$.

Bortel, R., Sovka, P., 2014. Approximation of the null distribution of the multiple coherence estimated with segment overlapping. Signal Processing 96, 310-314.

Boto, E., Holmes, N., Leggett, J., Roberts, G., Shah, V., Meyer, S.S., Muñoz, L.D., Mullinger, K.J., Tierney, T.M., Bestmann, S., Barnes, G.R., Bowtell, R., Brookes, M.J., 2018. Moving magnetoencephalography towards real-world applications with a wearable system. Nature. https://doi.org/10.1038/nature26147

Bourguignon, M., De Tiège, X., de Beeck, M.O., Ligot, N., Paquier, P., Van Bogaert, P., Goldman, S., Hari, R., Jousmäki, V., 2013. The pace of prosodic phrasing couples the listener's cortex to the reader's voice. Hum. Brain Mapp. 34, 314-326.

Bourguignon, M., Jousmäki, V., Op de Beeck, M., Van Bogaert, P., Goldman, S., De Tiège, X., 2012. Neuronal network coherent with hand kinematics during fast repetitive hand movements. Neuroimage 59, 1684-1691.

Bourguignon, M., Molinaro, N., Wens, V., 2017. Contrasting functional imaging parametric maps: The mislocation problem and alternative solutions. Neuroimage 169, 200-211.

Bourguignon, M., Piitulainen, H., De Tiège, X., Jousmäki, V., Hari, R., 2015.

Corticokinematic coherence mainly reflects movement-induced proprioceptive feedback. Neuroimage 106, 382-390.

Bourguignon, M., Whitmarsh, S., Piitulainen, H., Hari, R., Jousmäki, V., Lundqvist, D., 2016. Reliable recording and analysis of MEG-based corticokinematic coherence in the presence of strong magnetic artifacts. Clin. Neurophysiol. 127, 1460-1469.

Broderick, M.P., Anderson, A.J., Di Liberto, G.M., Crosse, M.J., Lalor, E.C., 2017. Electrophysiological correlates of semantic dissimilarity reflect the comprehension of natural, narrative speech. Current Biology 28, 803-809.

Carrette, E., De Tiège, X., Op De Beeck, M., De Herdt, V., Meurs, A., Legros, B., Raedt, R., Deblaere, K., Van Roost, D., Bourguignon, M., Goldman, S., Boon, P., Van Bogaert, P., Vonck, K., 2011a. Magnetoencephalography in epilepsy patients carrying a vagus nerve stimulator. Epilepsy Res. 93, 44-52.

Carrette, E., Op de Beeck, M., Bourguignon, M., Boon, P., Vonck, K., Legros, B., Goldman, S., Van Bogaert, P., De Tiège, X., 2011b. Recording temporal lobe epileptic activity with MEG in a light-weight magnetic shield. Seizure 20, 414-418.

Cencen, V., Hirotani, M., Chan, A.D.C., 2016. Comparison of active and passive electrodes in their optimized electroencephalography amplifier system, in: 2016 IEEE EMBS International Student Conference (ISC). https://doi.org/10.1109/embsisc.2016.7508603

Crosse, M.J., Di Liberto, G.M., Bednar, A., Lalor, E.C., 2016. The Multivariate Temporal Response Function (mTRF) Toolbox: A MATLAB Toolbox for Relating Neural Signals to Continuous Stimuli. Front. Hum. Neurosci. 10, 604.

Crowley, K.E., Colrain, I.M., 2004. A review of the evidence for P2 being an independent component process: age, sleep and modality. Clin. Neurophysiol. 115, 732-744. 
Dale, A.M., Sereno, M.I., 1993. Improved Localizadon of Cortical Activity by Combining EEG and MEG with MRI Cortical Surface Reconstruction: A Linear Approach. J. Cogn. Neurosci. 5, 162-176.

De Tiège, X., Carrette, E., Legros, B., Vonck, K., Op de Beeck, M., Bourguignon, M., Massager, N., David, P., Van Roost, D., Meurs, A., Lapere, S., Deblaere, K., Goldman, S., Boon, P., Van Bogaert, P., 2012. Clinical added value of magnetic source imaging in the presurgical evaluation of refractory focal epilepsy. J. Neurol. Neurosurg. Psychiatry $83,417-423$.

De Tiège, X., Op de Beeck, M., Funke, M., Legros, B., Parkkonen, L., Goldman, S., Van Bogaert, P., 2008. Recording epileptic activity with MEG in a light-weight magnetic shield. Epilepsy Res. 82, 227-231.

Di Liberto, G.M., O’Sullivan, J.A., Lalor, E.C., 2015. Low-Frequency Cortical Entrainment to Speech Reflects Phoneme-Level Processing. Curr. Biol. 25, 2457-2465.

Ding, N., Melloni, L., Zhang, H., Tian, X., Poeppel, D., 2016. Cortical tracking of hierarchical linguistic structures in connected speech. Nat. Neurosci. 19, 158-164.

Ding, N., Patel, A.D., Chen, L., Butler, H., Luo, C., Poeppel, D., 2017. Temporal modulations in speech and music. Neurosci. Biobehav. Rev. 81, 181-187.

Ding, N., Simon, J.Z., 2014. Cortical entrainment to continuous speech: functional roles and interpretations. Front. Hum. Neurosci. 8, 311.

Ding, N., Simon, J.Z., 2013. Adaptive temporal encoding leads to a background-insensitive cortical representation of speech. J. Neurosci. 33, 5728-5735.

Ding, N., Simon, J.Z., 2012. Emergence of neural encoding of auditory objects while listening to competing speakers. Proc. Natl. Acad. Sci. U. S. A. 109, 11854-11859.

Duez, L., Beniczky, S., Tankisi, H., Hansen, P.O., Sidenius, P., Sabers, A., Fuglsang-Frederiksen, A., 2016. Added diagnostic value of magnetoencephalography (MEG) in patients suspected for epilepsy, where previous, extensive EEG workup was unrevealing. Clin. Neurophysiol. 127, 3301-3305.

Efron, B., 1979. Bootstrap Methods: Another Look at the Jackknife. Ann. Stat. 7, 1-26.

Faes, L., Pinna, G.D., Porta, A., Maestri, R., Nollo, G., 2004. Surrogate data analysis for assessing the significance of the coherence function. IEEE Trans. Biomed. Eng. 51, 1156-1166.

Fuglsang, S.A., Dau, T., Hjortkjær, J., 2017. Noise-robust cortical tracking of attended speech in real-world acoustic scenes. Neuroimage 156, 435-444.

Giordano, B.L., Ince, R.A.A., Gross, J., Panzeri, S., Schyns, P.G., Kayser, C., 2016. Contributions of local speech encoding and functional connectivity to audio-visual speech integration. https://doi.org/10.1101/097493

Goldenholz, D.M., Ahlfors, S.P., Hämäläinen, M.S., Sharon, D., Ishitobi, M., Vaina, L.M., Stufflebeam, S.M., 2009. Mapping the signal-to-noise-ratios of cortical sources in magnetoencephalography and electroencephalography. Hum. Brain Mapp. 30, 1077-1086.

Gramfort, A., Luessi, M., Larson, E., Engemann, D.A., Strohmeier, D., Brodbeck, C., Parkkonen, L., Hämäläinen, M.S., 2014. MNE software for processing MEG and EEG data. Neuroimage 86, 446-460.

Gross, J., Hoogenboom, N., Thut, G., Schyns, P., Panzeri, S., Belin, P., Garrod, S., 2013. Speech rhythms and multiplexed oscillatory sensory coding in the human brain. PLoS Biol. 11, e1001752.

Halliday, D.M., Rosenberg, J.R., Amjad, A.M., Breeze, P., Conway, B.A., Farmer, S.F., 1995. A framework for the analysis of mixed time series/point process data-Theory 
and application to the study of physiological tremor, single motor unit discharges and electromyograms. Prog. Biophys. Mol. Biol. 64, 237-278.

Hämäläinen, M., Hari, R., 2002. Magnetoencephalographic Characterization of Dynamic Brain Activation: Basic Principles and Methods of Data Collection and Source Analysis, in: Brain Mapping: The Methods. pp. 227-253.

Hämäläinen, M., Hari, R., Ilmoniemi, R.J., Knuutila, J., Lounasmaa, O.V., $1993 a$. Magnetoencephalography - theory, instrumentation, and applications to noninvasive studies of the working human brain. Rev. Mod. Phys. 65, 413-497.

Hämäläinen, M., Hari, R., Ilmoniemi, R.J., Knuutila, J., Lounasmaa, O.V., 1993 b. Magnetoencephalography - theory, instrumentation, and applications to noninvasivee studies of the working human brain. Rev. Mod. Phys. 65, 413-497.

Hämäläinen, M.S., Ilmoniemi, R.J., 1994. Interpreting magnetic fields of the brain: minimum norm estimates. Med. Biol. Eng. Comput. 32, 35-42.

Hämäläinen, M.S., Lin, F.-H., Mosher, J.C., 2010. Anatomically and Functionally Constrained Minimum-Norm Estimates, in: MEG: An Introduction to Methods. pp. $186-215$.

Hari, R., Joutsiniemi, S.-L., Sarvas, J., 1988. Spatial resolution of neuromagnetic records: theoretical calculations in a spherical model. Electroencephalography and Clinical Neurophysiology/Evoked Potentials Section 71, 64-72.

Hari, R., Puce, A., 2017. MEG-EEG Primer. Oxford University Press.

Hillebrand, A., Fazio, P., de Munck, J.C., van Dijk, B.W., 2013. Feasibility of clinical magnetoencephalography (MEG) functional mapping in the presence of dental artefacts. Clin. Neurophysiol. 124, 107-113.

Hoen, M., Meunier, F., Grataloup, C.-L., Pellegrino, F., Grimault, N., Perrin, F., Perrot, X., Collet, L., 2007. Phonetic and lexical interferences in informational masking during speech-in-speech comprehension. Speech Commun. 49, 905-916.

Horton, C., D'Zmura, M., Srinivasan, R., 2013. Suppression of competing speech through entrainment of cortical oscillations. J. Neurophysiol. 109, 3082-3093.

Hotelling, H., 1931. The Generalization of Student's Ratio. Ann. Math. Stat. 2, 360-378.

Huiskamp, G., 1990. Difference formulas for the surface Laplacian on a triangulated surface. J. Comput. Phys. 91, 252-253.

Iwasaki, M., Pestana, E., Burgess, R.C., Lüders, H.O., Shamoto, H., Nakasato, N., 2005. Detection of epileptiform activity by human interpreters: blinded comparison between electroencephalography and magnetoencephalography. Epilepsia 46, 59-68.

Keitel, A., Gross, J., Kayser, C., 2018. Perceptually relevant speech tracking in auditory and motor cortex reflects distinct linguistic features. PLoS Biol. 16, e2004473.

Knake, S., Halgren, E., Shiraishi, H., Hara, K., Hamer, H.M., Grant, P.E., Carr, V.A., Foxe, D., Camposano, S., Busa, E., Witzel, T., Hämäläinen, M.S., Ahlfors, S.P., Bromfield, E.B., Black, P.M., Bourgeois, B.F., Cole, A.J., Cosgrove, G.R., Dworetzky, B.A., Madsen, J.R., Larsson, P.G., Schomer, D.L., Thiele, E.A., Dale, A.M., Rosen, B.R., Stufflebeam, S.M., 2006. The value of multichannel MEG and EEG in the presurgical evaluation of 70 epilepsy patients. Epilepsy Res. 69, 80-86.

Knappe, S.A., Sander, T.H., Trahms, L., 2014. Optically-Pumped Magnetometers for MEG, in: Magnetoencephalography: From Signals to Dynamic Cortical Networks. Springer.

Knowlton, R.C., 2008. Can magnetoencephalography aid epilepsy surgery? Epilepsy Curr. 8, $1-5$.

Knowlton, R.C., 2006. The role of FDG-PET, ictal SPECT, and MEG in the epilepsy surgery evaluation. Epilepsy Behav. 8, 91-101. 
Kösem, A., van Wassenhove, V., 2016. Distinct contributions of low- and high-frequency neural oscillations to speech comprehension. Language, Cognition and Neuroscience 32, 536-544.

Lalor, E.C., Foxe, J.J., 2010. Neural responses to uninterrupted natural speech can be extracted with precise temporal resolution. Eur. J. Neurosci. 31, 189-193.

Laszlo, S., Ruiz-Blondet, M., Khalifian, N., Chu, F., Jin, Z., 2014. A direct comparison of active and passive amplification electrodes in the same amplifier system. J. Neurosci. Methods 235, 298-307.

Laureys, S., Owen, A.M., Schiff, N.D., 2004. Brain function in coma, vegetative state, and related disorders. Lancet Neurol. 3, 537-546.

Legget, K.T., Hild, A.K., Steinmetz, S.E., Simon, S.T., Rojas, D.C., 2017. MEG and EEG demonstrate similar test-retest reliability of the $40 \mathrm{~Hz}$ auditory steady-state response. Int. J. Psychophysiol. 114, 16-23.

Luo, H., Poeppel, D., 2007. Phase patterns of neuronal responses reliably discriminate speech in human auditory cortex. Neuron 54, 1001-1010.

Mäkelä, J.P., Taulu, S., Pohjola, J., Ahonen, A., Pekkonen, E., 2007. Effects of subthalamic nucleus stimulation on spontaneous sensorimotor MEG activity in a Parkinsonian patient. Int. Congr. Ser. 1300, 345-348.

Medvedovsky, M., Taulu, S., Bikmullina, R., Ahonen, A., Paetau, R., 2009. Fine tuning the correlation limit of spatio-temporal signal space separation for magnetoencephalography. J. Neurosci. Methods 177, 203-211.

Mesgarani, N., Chang, E.F., 2012. Selective cortical representation of attended speaker in multi-talker speech perception. Nature 485, 233-236.

Meyer, L., Gumbert, M., 2018. Synchronization of Electrophysiological Responses with Speech Benefits Syntactic Information Processing. J. Cogn. Neurosci. 1-10.

Meyer, L., Henry, M.J., Gaston, P., Schmuck, N., Friederici, A.D., 2016. Linguistic Bias Modulates Interpretation of Speech via Neural Delta-Band Oscillations. Cereb. Cortex. https://doi.org/10.1093/cercor/bhw228

Molinaro, N., Lizarazu, M., Lallier, M., Bourguignon, M., Carreiras, M., 2016. Out-of-synchrony speech entrainment in developmental dyslexia. Hum. Brain Mapp. 37, 2767-2783.

Müller, J.A., Kollmeier, B., Debener, S., Brand, T., 2018. Influence of auditory attention on sentence recognition captured by the neural phase. Eur. J. Neurosci. https://doi.org/10.1111/ejn.13896

Ng, B.S.W., Logothetis, N.K., Kayser, C., 2013. EEG phase patterns reflect the selectivity of neural firing. Cereb. Cortex 23, 389-398.

Nichols, T.E., Holmes, A.P., 2001. Nonparametric permutation tests for functional neuroimaging: A primer with examples. Hum. Brain Mapp. 15, 1-25.

Oldfield, R.C., 1971. The assessment and analysis of handedness: the Edinburgh inventory. Neuropsychologia 9, 97-113.

Oostendorp, T.F., van Oosterom, A., 1996. The surface Laplacian of the potential: theory and application. IEEE Trans. Biomed. Eng. 43, 394-405.

Oostenveld, R., Fries, P., Maris, E., Schoffelen, J.-M., 2011. FieldTrip: Open source software for advanced analysis of MEG, EEG, and invasive electrophysiological data. Comput. Intell. Neurosci. 2011, 156869.

O’Sullivan, J.A., Power, A.J., Mesgarani, N., Rajaram, S., Foxe, J.J., Shinn-Cunningham, B.G., Slaney, M., Shamma, S.A., Lalor, E.C., 2014. Attentional Selection in a Cocktail Party Environment Can Be Decoded from Single-Trial EEG. Cereb. Cortex 25, 
$1697-1706$.

Owen, A.M., Coleman, M.R., Boly, M., Davis, M.H., Laureys, S., Pickard, J.D., 2006.

Detecting awareness in the vegetative state. Science 313, 1402.

Paulini, A., Fischer, M., Scheler, G., Hopfengärtner, R., Rampp, S., Kaltenhäuser, M., Dörfler, A., Buchfelder, M., Romstöck, J., Stefan, H., 2007. Lobar localization in epilepsy patients: Comparison of EEG and MEG. Int. Congr. Ser. 1300, 677-680.

Peelle, J.E., Gross, J., Davis, M.H., 2013. Phase-locked responses to speech in human auditory cortex are enhanced during comprehension. Cereb. Cortex 23, 1378-1387.

Pellegrino, F., Coupé, C., Marsico, E., 2011. Across-Language Perspective on Speech Information Rate. Language 87, 539-558.

Pereira, D.R., Cardoso, S., Ferreira-Santos, F., Fernandes, C., Cunha-Reis, C., Paiva, T.O., Almeida, P.R., Silveira, C., Barbosa, F., Marques-Teixeira, J., 2014. Effects of inter-stimulus interval (ISI) duration on the N1 and P2 components of the auditory event-related potential. Int. J. Psychophysiol. 94, 311-318.

Pernier, J., Perrin, F., Bertrand, O., 1988. Scalp current density fields: concept and properties. Electroencephalogr. Clin. Neurophysiol. 69, 385-389.

Perrin, F., Pernier, J., Bertrand, O., Echallier, J.F., 1989. Spherical splines for scalp potential and current density mapping. Electroencephalogr. Clin. Neurophysiol. 72, 184-187.

Petersen, E.B., Wöstmann, M., Obleser, J., Lunner, T., 2017. Neural tracking of attended versus ignored speech is differentially affected by hearing loss. J. Neurophysiol. 117, $18-27$.

Puschmann, S., Steinkamp, S., Gillich, I., Mirkovic, B., Debener, S., Thiel, C.M., 2017. The Right Temporoparietal Junction Supports Speech Tracking During Selective Listening: Evidence from Concurrent EEG-fMRI. J. Neurosci. 37, 11505-11516.

Reuter, M., Schmansky, N.J., Rosas, H.D., Fischl, B., 2012. Within-subject template estimation for unbiased longitudinal image analysis. Neuroimage 61, 1402-1418.

Riecke, L., Formisano, E., Sorger, B., Başkent, D., Gaudrain, E., 2018. Neural Entrainment to Speech Modulates Speech Intelligibility. Curr. Biol. 28, 161-169.e5.

Rimmele, J.M., Golumbic, E.Z., Schröger, E., Poeppel, D., 2015. The effects of selective attention and speech acoustics on neural speech-tracking in a multi-talker scene. Cortex $68,144-154$.

Rosen, S., 1992. Temporal information in speech: acoustic, auditory and linguistic aspects. Philos. Trans. R. Soc. Lond. B Biol. Sci. 336, 367-373.

Sekihara, K., Sahani, M., Nagarajan, S.S., 2005. Localization bias and spatial resolution of adaptive and non-adaptive spatial filters for MEG source reconstruction. Neuroimage $25,1056-1067$.

Shahin, A.J., Roberts, L.E., Miller, L.M., McDonald, K.L., Alain, C., 2007. Sensitivity of EEG and MEG to the N1 and P2 auditory evoked responses modulated by spectral complexity of sounds. Brain Topogr. 20, 55-61.

Simon, J.Z., 2015. The encoding of auditory objects in auditory cortex: insights from magnetoencephalography. Int. J. Psychophysiol. 95, 184-190.

Simpson, S.A., Cooke, M., 2005. Consonant identification in N-talker babble is a nonmonotonic function of N. J. Acoust. Soc. Am. 118, 2775-2778.

Stefan, H., Rampp, S., Knowlton, R.C., 2011. Magnetoencephalography adds to the surgical evaluation process. Epilepsy Behav. 20, 172-177.

Tallon-Baudry, C., Bertrand, O., Delpuech, C., Pernier, J., 1996. Stimulus specificity of phase-locked and non-phase-locked $40 \mathrm{~Hz}$ visual responses in human. J. Neurosci. 16, 4240-4249. 
Taulu, S., Hari, R., 2009. Removal of magnetoencephalographic artifacts with temporal signal-space separation: demonstration with single-trial auditory-evoked responses. Hum. Brain Mapp. 30, 1524-1534.

Taulu, S., Simola, J., 2006. Spatiotemporal signal space separation method for rejecting nearby interference in MEG measurements. Phys. Med. Biol. 51, 1759-1768.

Taulu, S., Simola, J., Kajola, M., 2005. Applications of the signal space separation method. IEEE Trans. Signal Process. 53, 3359-3372.

Thorpe, S.G., Nunez, P.L., Srinivasan, R., 2007. Identification of wave-like spatial structure in the SSVEP: comparison of simultaneous EEG and MEG. Stat. Med. 26, 3911-3926.

Vander Ghinst, M., Ghinst, M.V., Bourguignon, M., Op de Beeck, M., Wens, V., Marty, B., Hassid, S., Choufani, G., Jousmäki, V., Hari, R., Van Bogaert, P., Goldman, S., De Tiège, X., 2016. Left Superior Temporal Gyrus Is Coupled to Attended Speech in a Cocktail-Party Auditory Scene. J. Neurosci. 36, 1596-1606.

Varoquaux, G., Raamana, P.R., Engemann, D.A., Hoyos-Idrobo, A., Schwartz, Y., Thirion, B., 2017. Assessing and tuning brain decoders: Cross-validation, caveats, and guidelines. Neuroimage 145, 166-179.

Wehner, D.T., Hämäläinen, M.S., Mody, M., Ahlfors, S.P., 2008. Head movements of children in MEG: quantification, effects on source estimation, and compensation. Neuroimage 40, 541-550.

Wens, V., Marty, B., Mary, A., Bourguignon, M., Op de Beeck, M., Goldman, S., Van Bogaert, P., Peigneux, P., De Tiège, X., 2015. A geometric correction scheme for spatial leakage effects in MEG/EEG seed-based functional connectivity mapping. Hum. Brain Mapp. 36, 4604-4621.

Wheless, J.W., Willmore, L.J., Breier, J.I., Kataki, M., Smith, J.R., King, D.W., Meador, K.J., Park, Y.D., Loring, D.W., Clifton, G.L., Baumgartner, J., Thomas, A.B., Constantinou, J.E., Papanicolaou, A.C., 1999. A comparison of magnetoencephalography, MRI, and V-EEG in patients evaluated for epilepsy surgery. Epilepsia 40, 931-941.

Wolters, C.H., Anwander, A., Tricoche, X., Weinstein, D., Koch, M.A., MacLeod, R.S., 2006. Influence of tissue conductivity anisotropy on EEG/MEG field and return current computation in a realistic head model: A simulation and visualization study using high-resolution finite element modeling. Neuroimage 30, 813-826.

Zion-Golumbic, E.M., Ding, N., Bickel, S., Lakatos, P., Schevon, C.A., McKhann, G.M., Goodman, R.R., Emerson, R., Mehta, A.D., Simon, J.Z., Poeppel, D., Schroeder, C.E., 2013. Mechanisms underlying selective neuronal tracking of attended speech at a “cocktail party." Neuron 77, 980-991.

Zion-Golumbic, E.M., Poeppel, D., Schroeder, C.E., 2012. Temporal context in speech processing and attentional stream selection: a behavioral and neural perspective. Brain Lang. 122, 151-161.

Zion-Golumbic, E., Schroeder, C.E., 2012. Attention modulates "speech-tracking" at a cocktail party. Trends Cogn. Sci. 16, 363-364. 


\section{Tables and figures}

\begin{tabular}{|c|c|c|c|c|c|c|c|c|c|c|c|c|}
\hline \multirow{4}{*}{ Condition } & \multicolumn{6}{|c|}{ delta } & \multicolumn{6}{|c|}{ theta } \\
\hline & \multicolumn{12}{|c|}{ Coherence-based speech-brain tracking values } \\
\hline & \multicolumn{2}{|l|}{ MEG } & \multicolumn{2}{|l|}{ EEG } & \multicolumn{2}{|l|}{ MEEG } & \multicolumn{2}{|l|}{ MEG } & \multicolumn{2}{|l|}{ EEG } & \multicolumn{2}{|l|}{ MEEG } \\
\hline & Mean \pm SD & $\mathrm{N}$ & Mean \pm SD & $\mathrm{N}$ & Mean $\pm S D$ & $\mathrm{~N}$ & Mean \pm SD & $\mathrm{N}$ & Mean $\pm S D$ & $\mathrm{~N}$ & Mean \pm SD & $\mathrm{N}$ \\
\hline noiseless & $0.1855 \pm 0.0677$ & 10 & $0.0574 \pm 0.021$ & 8 & & & $0.0233 \pm 0.0121$ & 8 & $0.0126 \pm 0.0041$ & $\frac{6}{6}$ & & \\
\hline noise & $0.1091 \pm 0.0455$ & 9 & $0.0533 \pm 0.0218$ & 7 & & & $0.0201 \pm 0.0093$ & 8 & $0.0121 \pm 0.004$ & 6 & & \\
\hline & \multicolumn{12}{|c|}{ Reconstruction accuracy based speech-brain tracking values } \\
\hline noiseless & $0.4079 \pm 0.1217$ & 10 & $0.2609 \pm 0.0971$ & 10 & $0.4164 \pm 0.1333$ & 10 & $0.1042 \pm 0.0427$ & 10 & $0.0615 \pm 0.0411$ & 9 & $0.1115 \pm 0.0472$ & 10 \\
\hline
\end{tabular}

Table 1. Mean $\pm \mathrm{SD}$ speech brain tracking values and number $\mathrm{N}$ of participants for whom they were statistically significant.

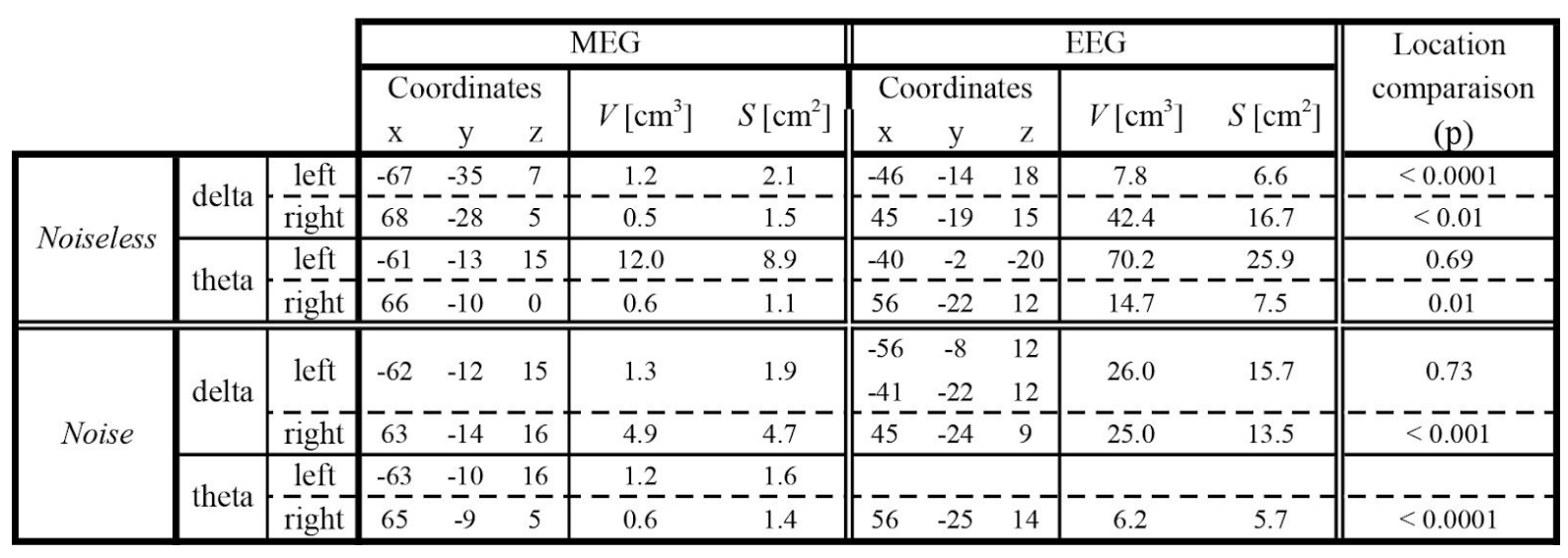

Table 2. MNI coordinates and confidence volume $(V)$ and surface $(S)$ of peak speech brain tracking, and $p$ value quantifying the significance of a difference in location between MEG and EEG.

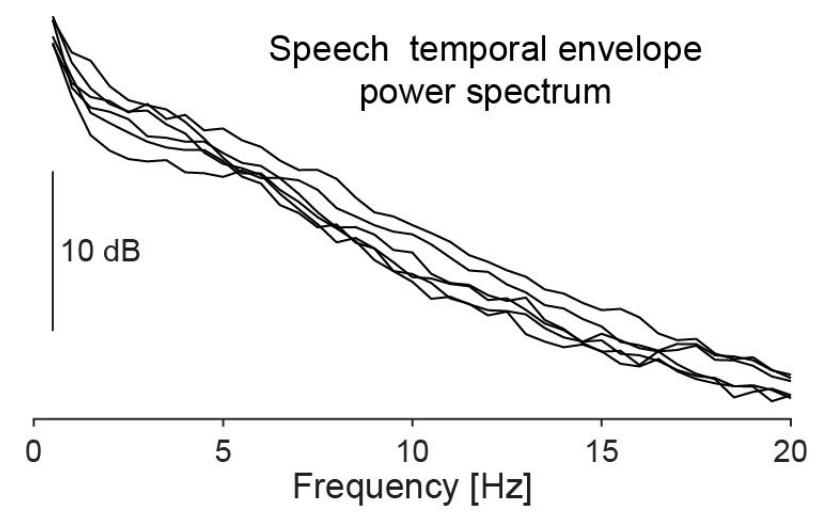


Figure 1. Power spectrum of speech temporal envelope for the 6 different audio recordings.
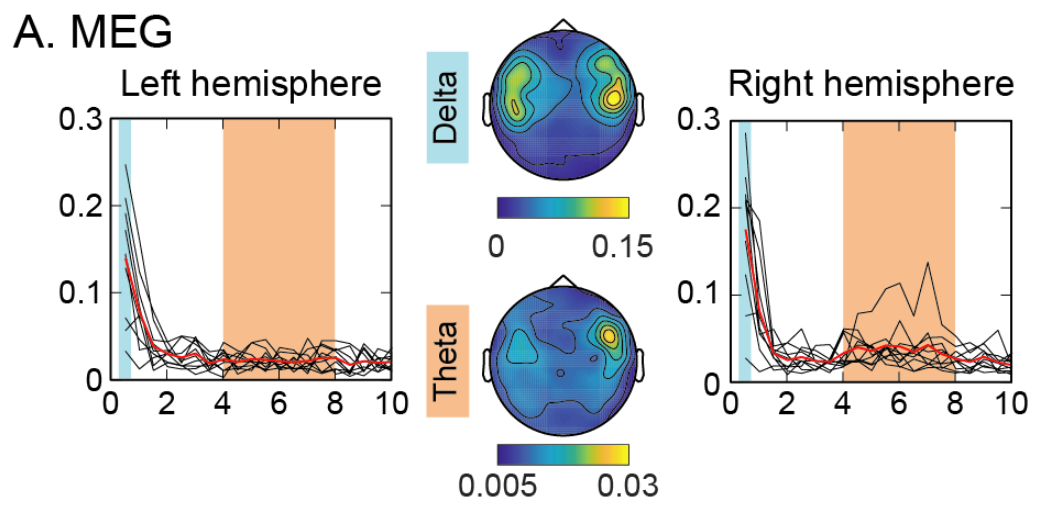

\section{B. EEG}

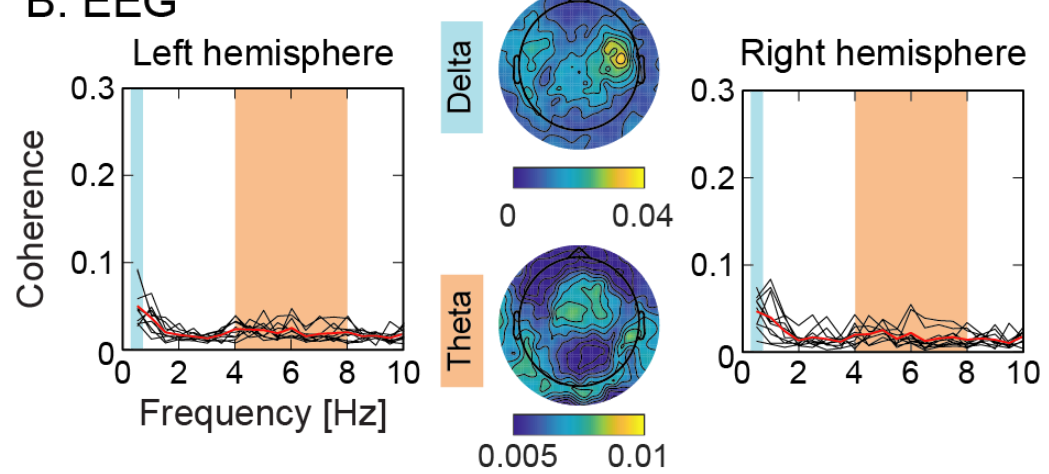

Figure 2. Speech brain tracking in the noiseless condition based on MEG (A) and EEG (B) recordings. Boxes on the left (respectively right) present coherence spectra computed between the attended speech stream and MEG/EEG signals from the left (respectively right) hemisphere. In each plot, there is one black trace per subject and group mean is in red. Between coherence spectra are the corresponding group-level spatial distributions at delta $(0.5 \mathrm{~Hz})$ and theta $(4-8 \mathrm{~Hz})$ frequencies. 

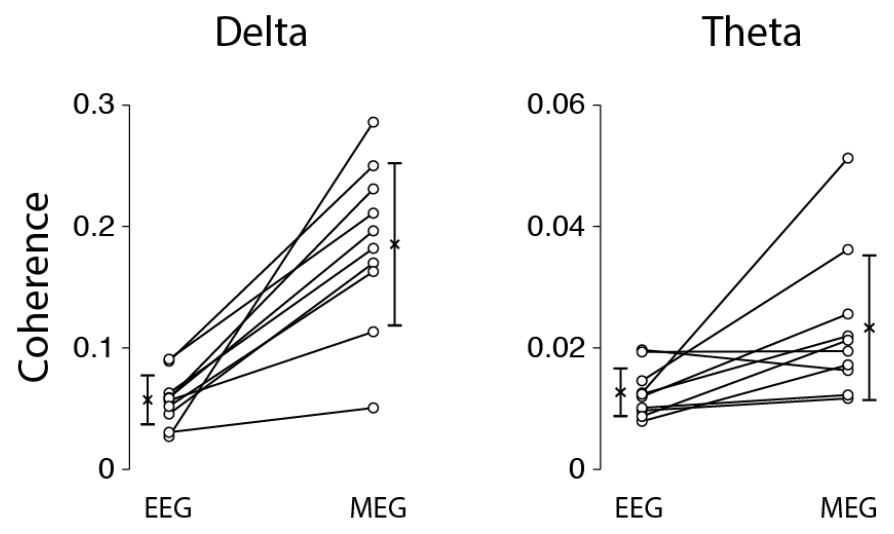

Figure 3. Coherence-based speech brain tracking values in noiseless condition. Circles provide individual coherence levels estimated based on MEG and EEG recordings at delta (left) and theta frequencies (right). Group mean and standard deviation are presented aside from individual values.
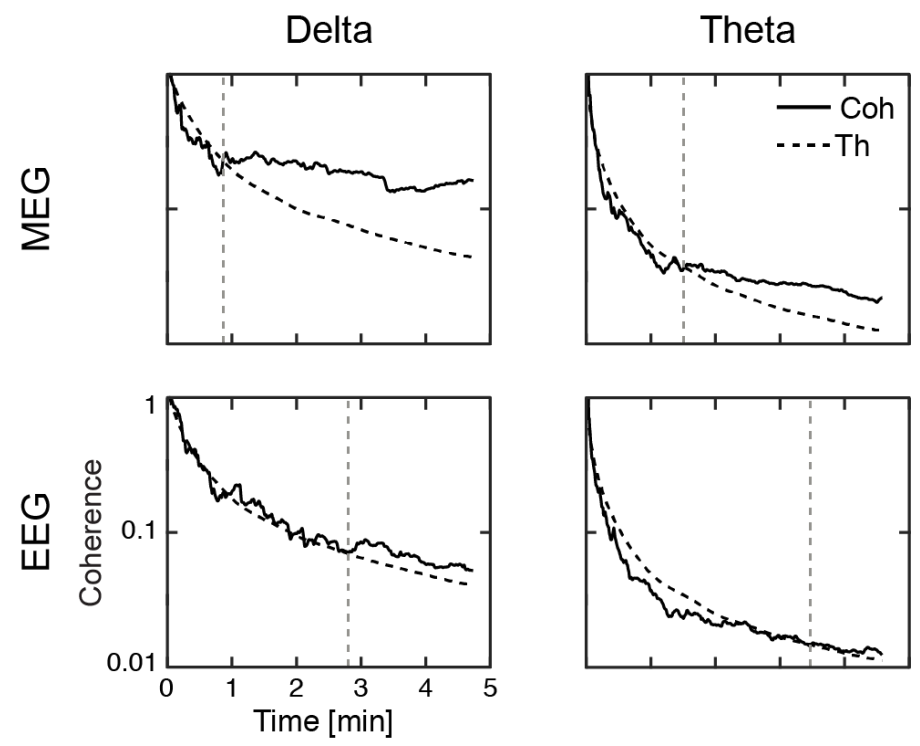

Figure 4. Minimum necessary recording time to obtain significant speech brain tracking with coherence analysis in the noiseless condition. Data from a representative subject (a different one for delta and theta frequencies) are presented here. Connected traces represent the 
maximum coherence across all channels (Coh) as function of the recording time. Dashed traces are the significance threshold at $p<0.05(\mathrm{Th})$, which also depend on the recording length. A gray vertical dashed line indicates the minimum necessary recording time, that is, the time point at which maximum coherence becomes and remains significant (i.e., the connected trace crosses and remains above the black dashed trace).
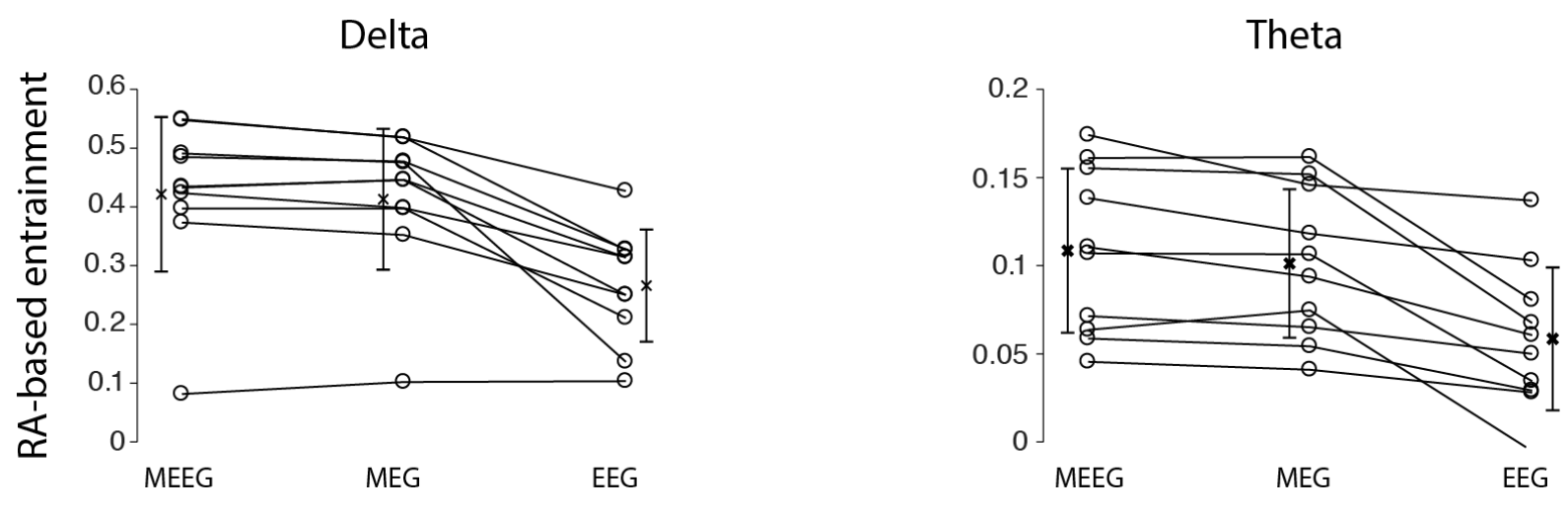

Figure 5. Same as Figure 3 for reconstruction accuracy (RA)-based speech brain tracking values in the noiseless condition. 
A. MEG
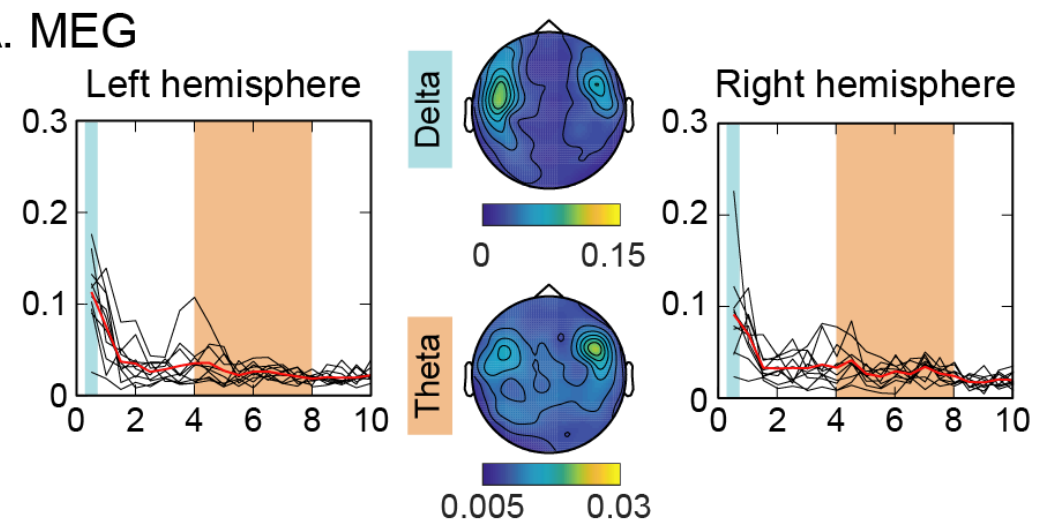

B. EEG
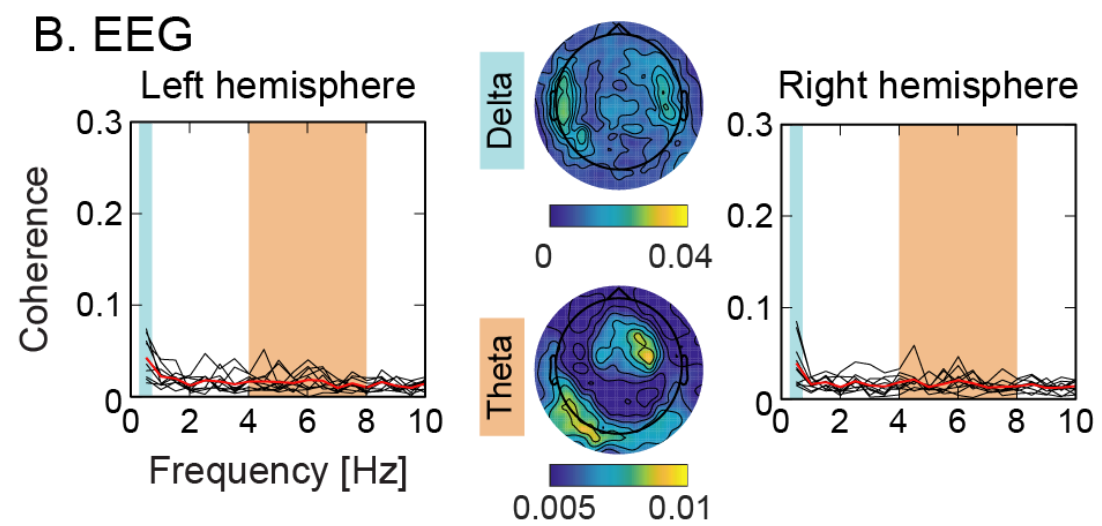

Figure 6. Same as Figure 2 for the noise condition.
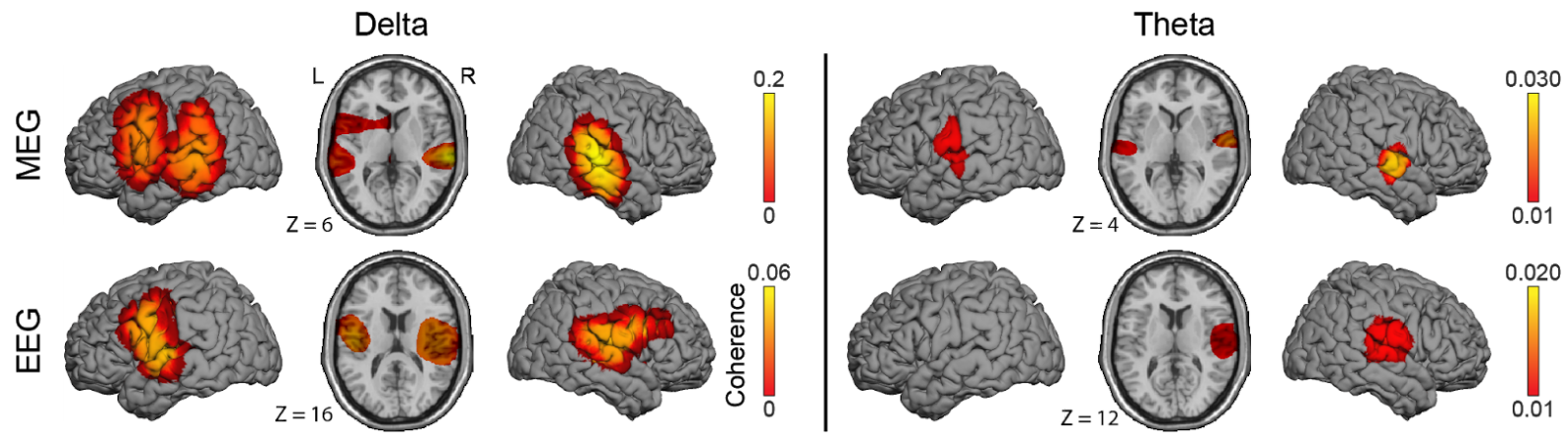

Figure 7. Group-level coherence maps of speech brain tracking in the noiseless condition. Maps are thresholded at statistical significance level, and different scales are used for MEG and EEG, and for maps at delta and theta frequencies, to match maps maximum value. Note that maximum coherence at theta frequencies reach higher values than those in scalp maps shown in Figs. 2 and 6. This is mainly due to that coherence at a given source was here 
optimized across 3 orientations, a procedure that inflates values especially when there is no significant coupling.
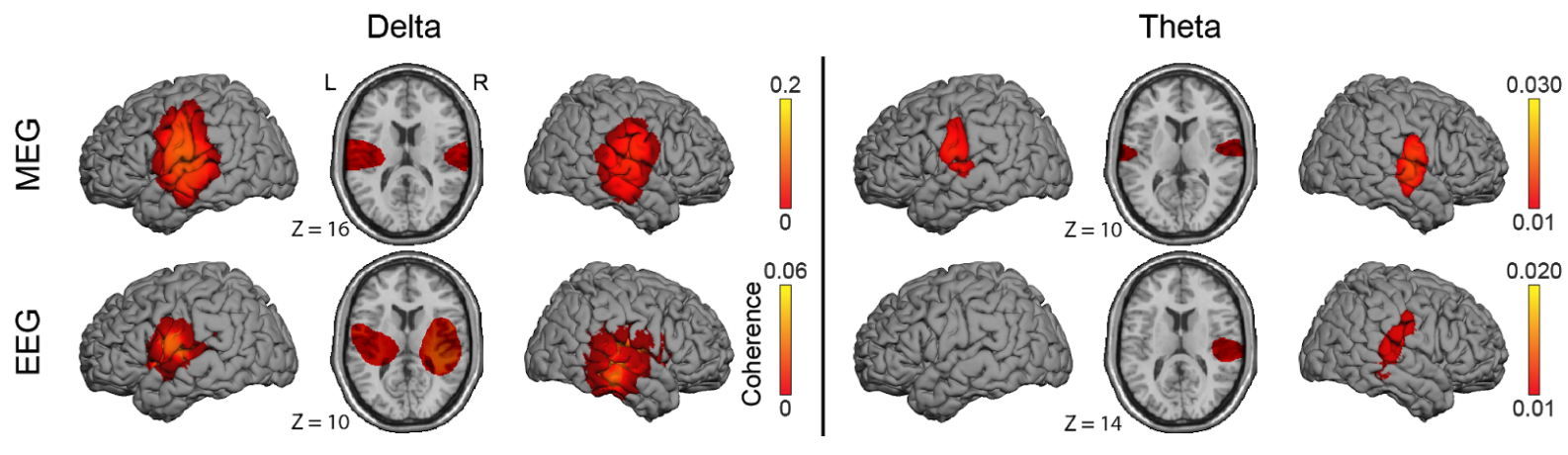

Figure 8. Same as Fig. 7 for the noise condition. 